\title{
Pressure Oscillations Caused by Momentum on Shut In of a High Rate Well in a Fractured Formation
}

\author{
by
Sanjay Bhatnagar \\ June 1989
}

A Report

Submitted to the Department of Petroleum Engineering, Stanford University In Partial Fulfillment of the Requirements

For the Degree of

Master of Science 
SGP-TR-1 $\div 9$

\title{
Pressure Oscillations Caused by Momentum on Shut In of a High Rate Well in a Fractured Formation
}

\author{
by \\ Sanjay Bhatnagar \\ June 1989
}

\begin{abstract}
A Report
Submitted to the Department of

Petroleum Engineering, Stanford University

In Partial Fulfillment of the Requirements

For the Degree of

Master of Science
\end{abstract}

\begin{abstract}
DISCLAIMER
This report was prepared as an account of work sponsored by an agency of the United States Government. Neither the United States Government nor any agency thereof, nor any of their employees, makes any warranty, express or implied, or assumes any legal liability or responsibility for the accuracy, completeness, or usefulness of any information, apparatus, product, or process disclosed, or represents that its use would not infringe privately owned rights. Reference herein to any specific commercial product, process, or service by trade name, trademark, manufacturer, or otherwise does not necessarily constitute or imply its endorsement, recummendation, or favoring by the United States Government or any agency thereof. The views and opinions of authors expressed herein do not necessarily state or reflect those of the United States Government or any agency thereof.
\end{abstract}




\section{Contents}

Acknowledgements $\quad$ iii

Table of Contents $\quad v$

List of Tables

List of Figures vii

1 Introduction 1

2 Problem Definition 3

3 Literature Review 10

4 Methodology for Solution $\quad 12$

5 Inertia of liquid in a wellbore $\quad 13$

5.1 Basic Equations . . . . . . . . . . . . . . . . . 13

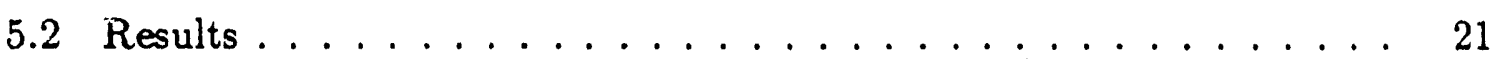

5.3 Effect of Transducer Tirne Constant on the Oscillations . . . . . . 23

6 Cartesian Solution $\quad 27$

6.1 Continuity Equation ........................ 27

6.2 Navie: Stokes Equation . . . . . . . . . . . . . . 30

6.3 Solution of Systern Equations ..................... 31

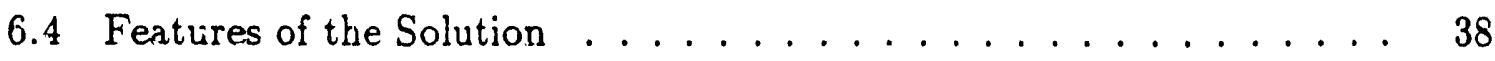


7 Future Work $\quad 39$

8 Symbols $\quad 41$

9 Appendix A $\quad 44$

9.1 Navier Stokes Equations . . . . . . . . . . . . . . . . . . . 44

9.1.1 Cartesian Co-ordinates . . . . . . . . . . . . . 44

9.1.2 Radial Co-ordinates.................. 45

9.2 Continuity Equation . . . . . . . . . . . . . . . 46

$\begin{array}{ll}10 \text { Appendix B } & \mathbf{4 7}\end{array}$

10.1 Continuity Equation . . . . . . . . . . . . . . 47

10.2 Navier Stokes Equations . . . . . . . . . . . . . . . . 48

10.3 Simultaneous Solution of System Equations . . . . . . . . . . . 49

$\begin{array}{ll}\text { Bibliography } & 55\end{array}$ 


\section{List of Tables}

2.1 Estimated parameter values for the Mexican well . . . . . . . . 8 


\section{List of Figures}

2.1 Pressure vs. time for pressure buildup in a Mexican oil well . . . . . 4

2.2 Mexican pressure buildup with expanded pressure scale . . . . . . 5

2.3 Mexican pressure buildup with expanded pressure and time scales . . 6

2.4 Spinner flow rate vs. time during Mexican pressure buildup . . . . . 7

5.1 Schematic diagram of well and reservoir . . . . . . . . . . . . 14

5.2 Pressure drop vs. time for a constant flow rate of $5000 \mathrm{~b} / \mathrm{d}$ for 1000 seconds and then shut in . . . . . . . . . . . . 22

5.3 Pressure vs. time for model and a transducer with a 0.1 second time

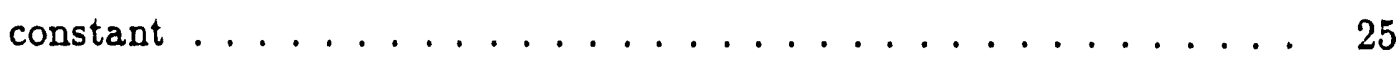

5.4 Pressure vs. time for model and a transducer with a 0.6 second time constant ..................... 26

6.1 Schematic of linear flow with inertia model . . . . . . . . . . 28

6.2 Schematic of a single fracture with inertia model . . . . . . . . . 29

6.3 Dimensionless pressure vs. dimensionless time for $\beta=1.0$ for a production time $t_{p_{D}}=10,000 \ldots \ldots \ldots \ldots \ldots$

6.4 Dimensionless pressure vs. dimensionless time for a transducer time constant of 0.5 seconds, $t_{p_{D}}=10,000 \ldots \ldots \ldots$ 


\section{Section 1}

\section{Introduction}

Pressure transient testing techniques are an important part of reservoir and production testing procedures. These techniques are frequently used to determine practical information about underground reservoirs such as the permeability, porosity, liquid content, reservoir and liquid discontinuities and other related data. This information is valuable in helping to analyze, improve and forecast reservoir performance.

A transient well test is performed by changing flow conditions in a wellbore and observing the pressure response. Monitoring of the pressure response may be done at the surface or maybe performed downhole. The data thus obtained is then analyzed by matching the response obtained during the well test with a solution provided by a mathematical, numerical or a physical model. The basic assumption underlying this approach is that the model accurately describes the reservoir behavior. Reservoir properties can be inferred from those used in the model to obtain the match.

The well flow rate maybe changed in a number of ways, and each leads to a different type of test. Whether a particular test can be analyzed or not depends upon the availability of a model to describe that particular test. It is thus important to develop models for the different types of well tests and for the i.ifferent types of reservoirs in which tests are conducted.

This report is concerned with developing models for pressure transient well testing in high permeability, high flow rate, naturally fractured reservoirs. Theoretical models presently available are inadequate for handling these well tests because they do not 
include the inertial effects of the liquid in the wellbore and the fractures. The basic assumptions commonly used in currently available theoretical models for naturally fractured reservoirs are:

1. mass conservation is assumed,

2. Darcy's Law applies in the matrix and the fractures,

3. it is assumed that the wellbore, fractures and the matrix are saturated by a single phase, slightly compressible liquid,

4. How from the fractures to the wellbore is much greater than the flow from the matrix to the wellbore,

5. inertial, gravitational and frictional effects are neglected,

6. turbulent flow in fractures and the fracture wellbore interface is neglected,

7. in the fracture network, isotropic permeabilities are assumed,

8. a fractured reservoir is assumed to consist of a regular array of matrix blocks, though recent work considers a reservoir as having blocks with some uniform distribution, and

9. skin and wellbore storage influence well pressure behavior.

Pressure behavior recently observed in several well tests conducted in high flow rate oil wells in Mexico exhibited oscillations, believed caused by the inertial effects of a liquid in the wellbore and perhaps formation fractures. In the present work, a study was made of the effects of liquid inertia in the fractures and the wellbore on the pressure response obtained during a well test. The effects of turbulent flow and multi-phase flow effects such as gravitational segregation or anisotropic porous media effects were not considered. The scope of the study was limited to studying inertial effects on the pressure response of a fractured reservoir. 


\section{Section 2}

\section{Problem Definition}

This study concerns some well tests performed in Mexico on extremely high flow rate oil wells in naturally fractured reservoirs. A typical example of a well test result is shown in Fig. 2.1. The figure shows a linear plot of Hewlett Packard (HP) gage pressure vs. elapsed time during a pressuce buildup test. The well was shut in at the wellhead and a simultaneous pressure and flowrate recording was made downhole. The data from the spinner (flow rate) and other plots showing the nature of the well test are presented in Figs. 2.2-2.4.

The following information is available on the well and formation:

1. matrix permeability is $0.1 \mathrm{md}$,

2. fracture permeability estimated at 1-10 darcy,

3. matrix porosity is 0.06 fraction bulk volume,

4. producing formation thickness is 40 meters,

5. mobile formation liquid is single phase oil of viscosity $0.5 \mathrm{cp}$ and a bubble point of $3,415 \mathrm{psi}$, and

6. oil is produced through packed off tubing. The tubing size is 7 inches. 

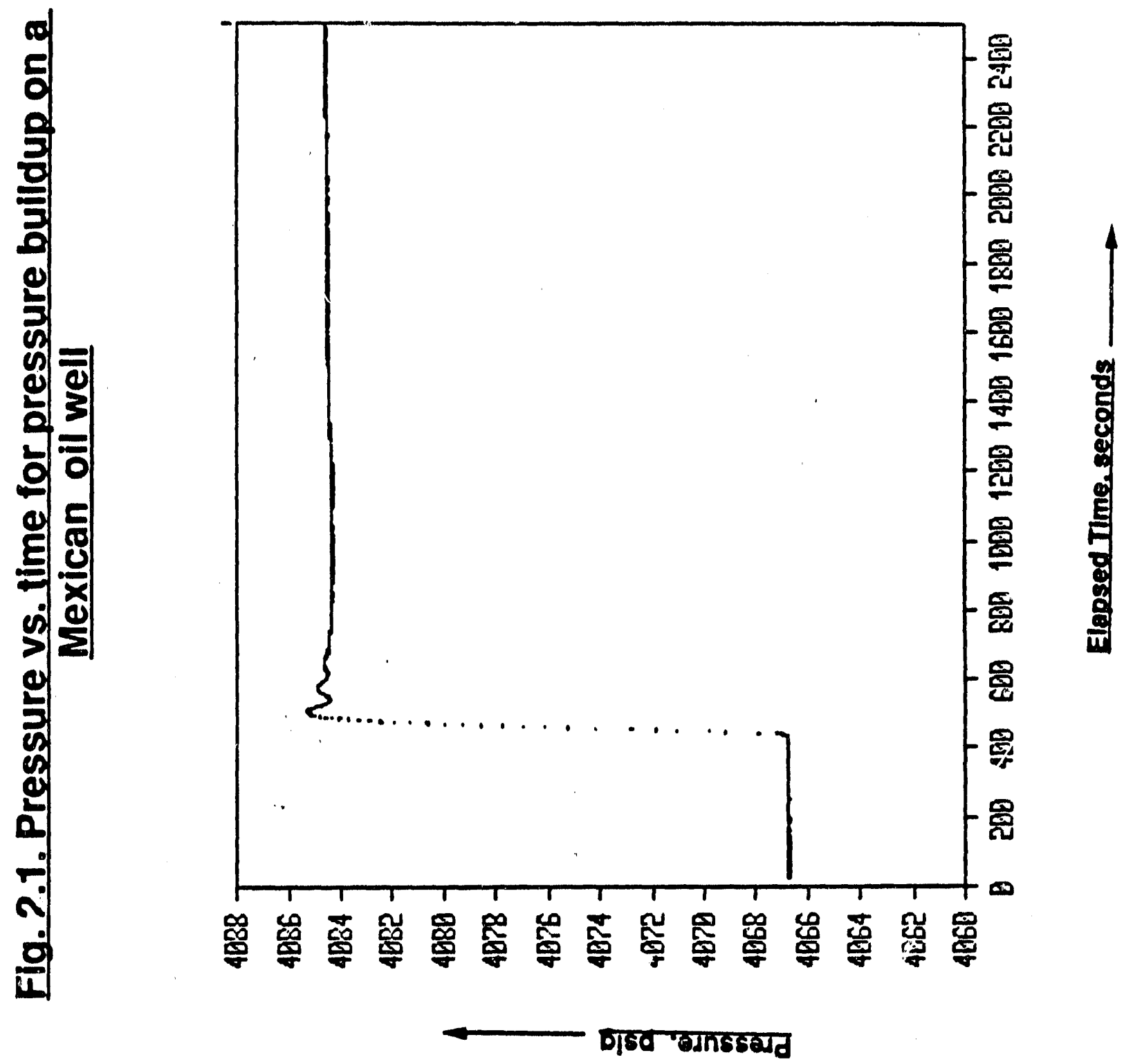


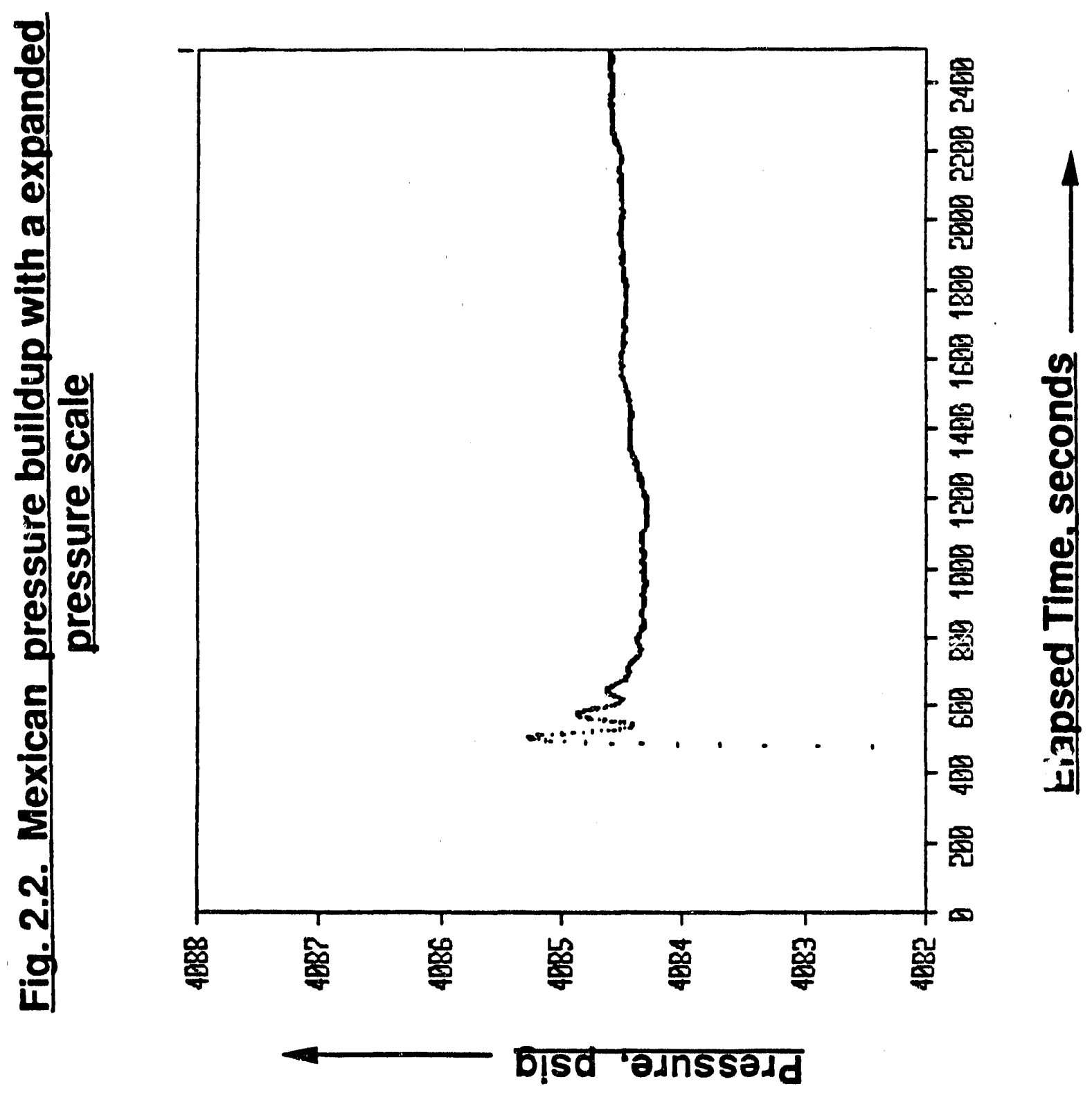




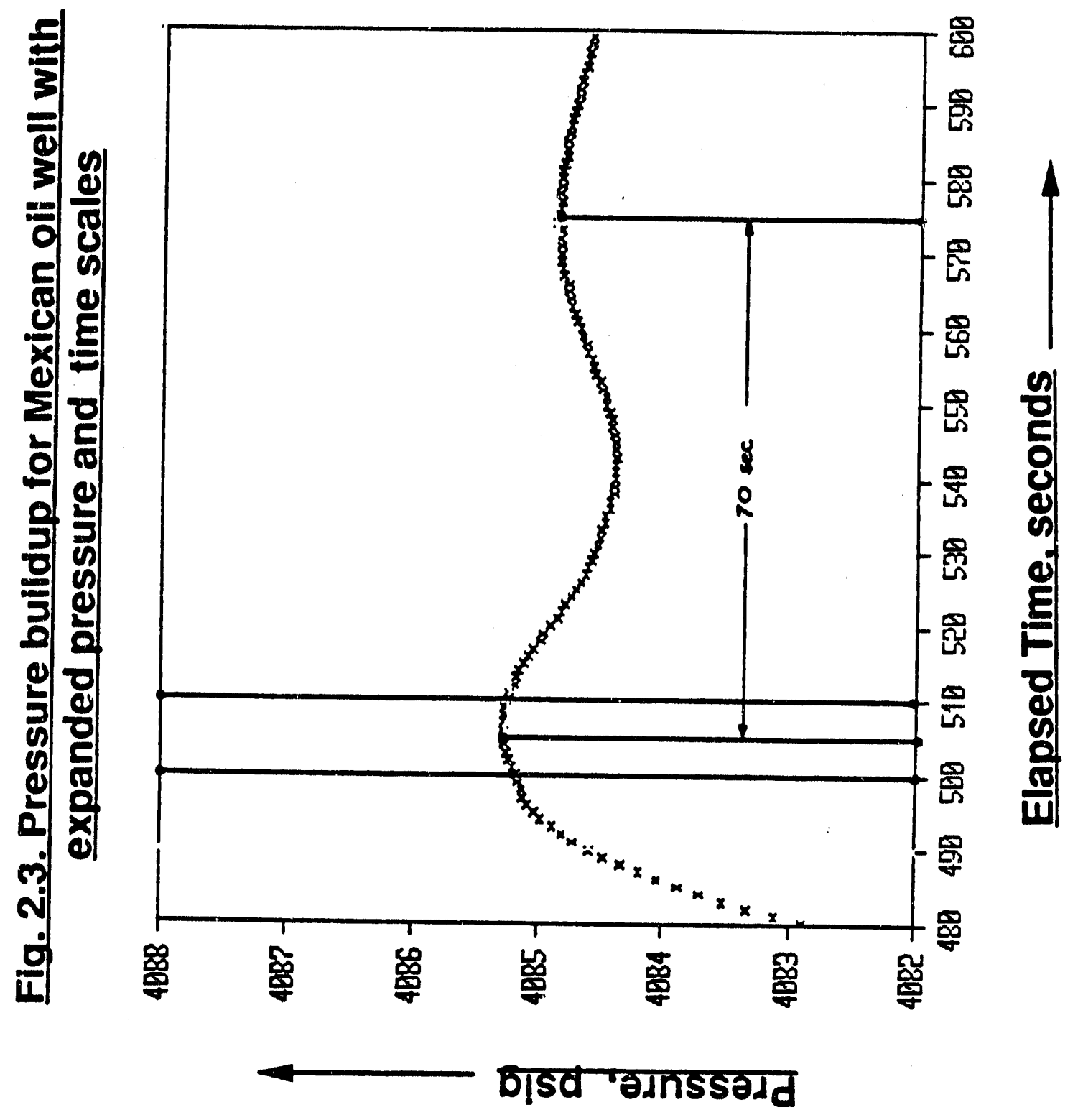




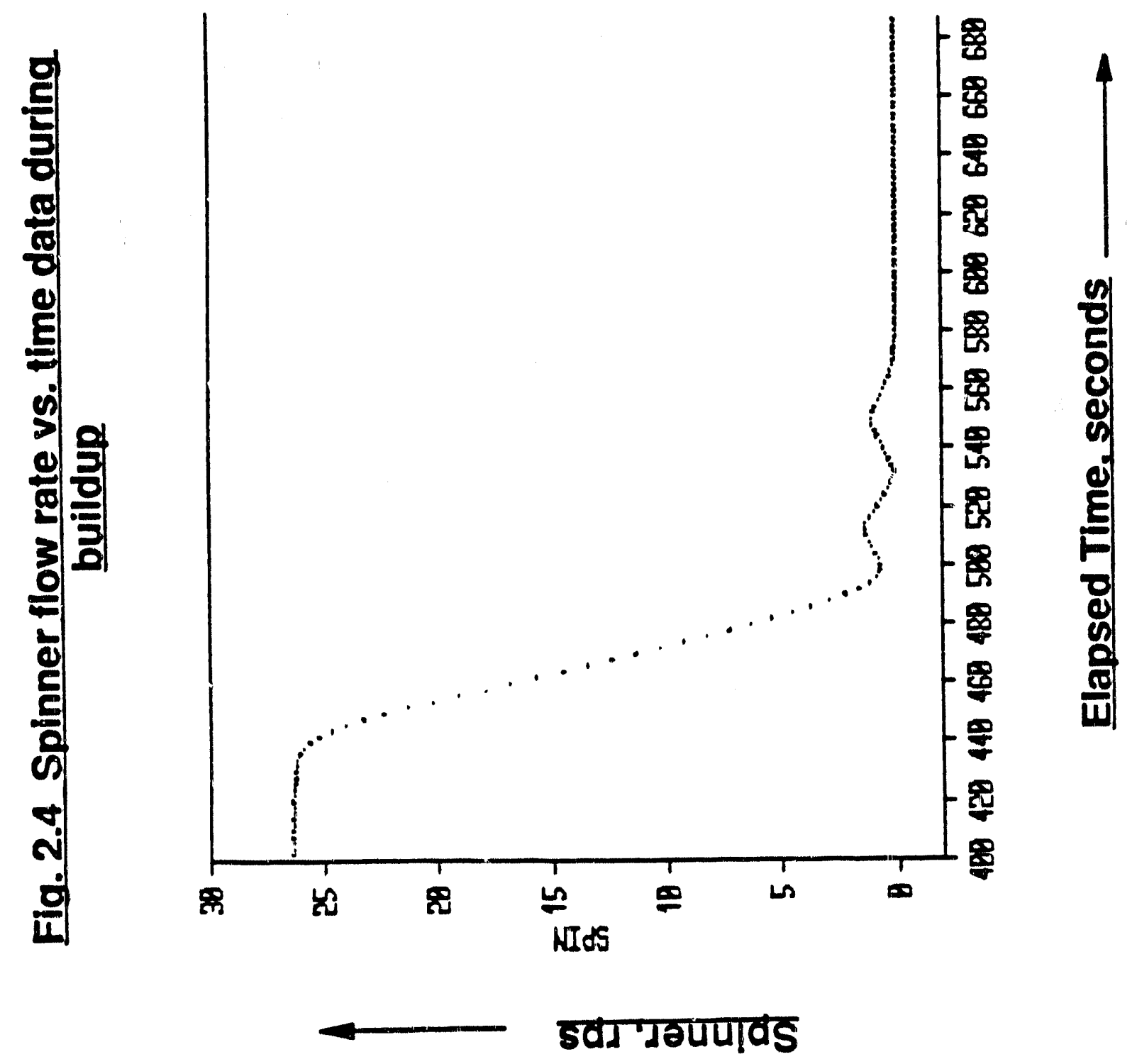




\begin{tabular}{|l|rl|}
\hline $\mathrm{q}$ & 25,000 & bbls/day \\
\hline $\mathrm{U}$ & 0.151 & meters $/ \mathrm{sec}$ \\
\hline $\mathrm{h}$ & $\approx 40$ & meters \\
\hline $\mathrm{H}$ & 3048 & meters \\
\hline$\rho$ & 800 & $\mathrm{~kg} / \mathrm{m}^{3}$ \\
\hline$\mu$ & 0.001 & pascal-second \\
\hline$\tau$ & 70 & seconds \\
\hline $\mathrm{k}$ & 1 & darcy \\
\hline
\end{tabular}

Table 2.1: Estimated parameter values for the Mexican wel!

The estimated parameters for the well may be summarised in Table 2.1.

The following observations may be made from the various data available for the well test:

1. the well flow rate before shut in was very higi, in the range of 25,000 barrels per day,

2. the pressure response shown in Fig. 2.1 is due to a step shut in, i.e the flow rate was rapidly dropped to zoro at the surface,

3. the pressure response shows underdamped oscillations with a time period of about 70 seconds(see Fig. 2.3),

4. the amplitude of the first pressure oscillation was of the order of one psi; later oscillations rapidly decay to a stable pressure reading,

5. the flowrate spinner response showed an oscillating flow entering and leaving the formation for the duration of the oscillations(see Fig. 2.4), and

6. the oscillations occurred for several minutes at the start of buildup and masked the early time storage and skin response.

For high production rate from fractured reservoirs, the kinetic energy of the liquid moving in the fractures and the wellbore may be important and contribute to the early time pressure response. The mass of liquid flowing to the borehole from the fractures 
and in the wellbore is decelerated rapidly during the well shutin (build up). This may cause a momentum effect at early times. This pressure effect propagates up and down the well bore and fractures until the energy is dissipated by friction. This is the premise of the work presented in this study. The relative importance of inertial effects in the wellbore and in formation fractures was also an objective of the study.

In the next chapter we consider literature on inertial effects. 


\section{Section 3}

\section{Literature Review}

In 1856 Darcy [7] stated the law of laminar flow through porous media. This law has been generalized to describe laminar mutiphase flow of oil, gas and water through reservoir rocks. Although originally empirical, Darcy's Law has been derived on a fundamental basis by assuming that inertial effects are negligible.

In 1965 Bredehoeft et al. [2] addressed the subject of oscillations in groundwater wells. They considered pressure oscillations in groundwater wells observed in response to an earthquake. They also considered the effect of inertia on a water column due to a small initial variation in liquid level, neglecting friction losses. In 1966 the authors [1]introduced wellbore storage into the problem description and developed several specific solutions. In 1967 Bredehoeft [3] described changes in wellbore liquid level in response to earth tides caused by the passage of the moon.

McMillen et al. [10] in 1967 considered inertial effects on flow in porous media in an early study. They concluded that the Telegraph Equation was more appropriate than the Diffusivity Equation. They did not consider a fractured porous medium.

In 1976, van der Kamp[20] proposed an approximate method to determine reservoir transmissivity by means of slug test data analysis for systems exhibiting an oscillatery response in the wellbore. This theory was based on the wellbore equation (incorporating a momentum balance) previously developed by Bredehoeft et al. in 1965.

In 1979, Shinohara and Ramey[17] extended van der Kamp's work on slug test data 
analysis by considering wellbore storage, skin effect and inertial effects on a wellbore liquid column. Saldana in his $1983 \mathrm{Ph} . \mathrm{D}$ dissertation [15] extended Shinohara's work. He considered wellbcie friction and iniproved the wellbore momentum balance.

In 1985 Holzhausen and Gooch [14] presented a method for determining the dimensions of hydraulic fractures by analyzing the time period of free pressure oscillations measured in a wellbore. The method was called hydraulic impedance analysis. They also considered the effect of liquid in the hydraulic fracture.

In 1986, Saldana and Ramey [16] presented a paper on wellbore inertial and frictional effects during slug tests and drill stem tests. The paper considered inertial effects in the wellbore, and coupled a momentum balance equation for the wellbore to the diffusivity equation for the reservoir. The resulting solution in laplace space was solved by numerical inversion using the Stehfest(1970) [18] algorithm. The authors presented criteria useful to evaluate the magnitude of inertial and frictional effects from the properties of the wellbore-reservoir system.

Guenther and Lundy [12] in 1986, presented a numerical treatment of the response of a well aquifer system to slug testing. The method used Green's functions to solve the coupled wellbore equation and the diffusivity equation governing the behavior of the system. The method considered inertial and frictional effects in the wellbore and compared the results of the solution with observed data.

Holzhausen and Egan [13] in 1986, applied the hydraulic impedance method for fracture evaluation to two tests in minifracture testing.

Although there is limited literature on inertial effects in well test analysis, there is evidence that such effects can cause the oscillations observed in the Mexican well tests. Holzhausen and Gooch, 1985, observed a definite change in the period of oscillations before and after hydraulic fracturing. The time period of the oscillations increased from about 1 second before fracturing to 2 seconds after fracturing. This suggested that natural formation fractures and /or the wellbore could explain the Mexican well test data. The method for solution of the Mexican well test problem is presented in the next chapter. 


\section{Section 4}

\section{Methodology for Solution}

Inertial effects for high flow rate liquid flow in naturally-fractured formations has not been studied. In view of the unusual pressure oscillations in the Mexican well test it was evident that a need existed for:

1. identifying the source of the oscillations,

2. developing a model for the well test, and

3. determining whether useful reservoir parameters could be determined by matching such a model with the available well test data.

In keeping with these objectives, it was decided to derive solutions for liquid inertia in the wellbore and liquid inertia in a fractured formation, for pressure buildup. Liquid inertia in the wellbore coupled with Darcy laminar liquid flow(Diffusivity Equation) in the reservoir was one model. The equations for the wellbore were derived from the Navier Stokes and Continuity equations. By using the Diffusivity Equation for the reservoir we neglected flow inertia in the reservoir. This model helped determine whether the cause of the pressure oscillations was inertia of liquid in the wellbore.

Another model considered inertial effects of liquid in a single fracture in the reservoir. The fracture was coupled to the wellbore, but the model did not consider inertial or gravitational effects in the wellbore. This was done to permit identification of the cause of pressure oscillations. To aid understanding, this model was first constructed for linear flow and then later extended to a more realistic radial flow system. 


\section{Section 5}

\section{Inertia of liquid in a wellbore}

In this section we consider the effects of the mass of liquid in a wellbore upon pressure in a wellbore, as flow is started and stopped.

\subsection{Basic Equations}

First, we will consider inertial effects in a wellbore. We start with the Continuity Equation:

$$
\frac{\partial \rho}{\partial t}+\frac{\partial}{\partial x}(\rho w)+\frac{\partial}{\partial y}(\rho v)+\frac{\partial}{\partial z}(\rho u)=0
$$

where $w, v$ and $u$ are velocities in the $x, y$ and the $z$ directions, respectively(Schematic in Fig. 5.1).We make the following assumptions:

$$
\begin{aligned}
& w=0 \\
& v=0
\end{aligned}
$$

The isothermal compressibility of a liquid is defined as:

$$
c=\frac{1}{\rho}\left(\frac{d \rho}{d p}\right)_{T}
$$




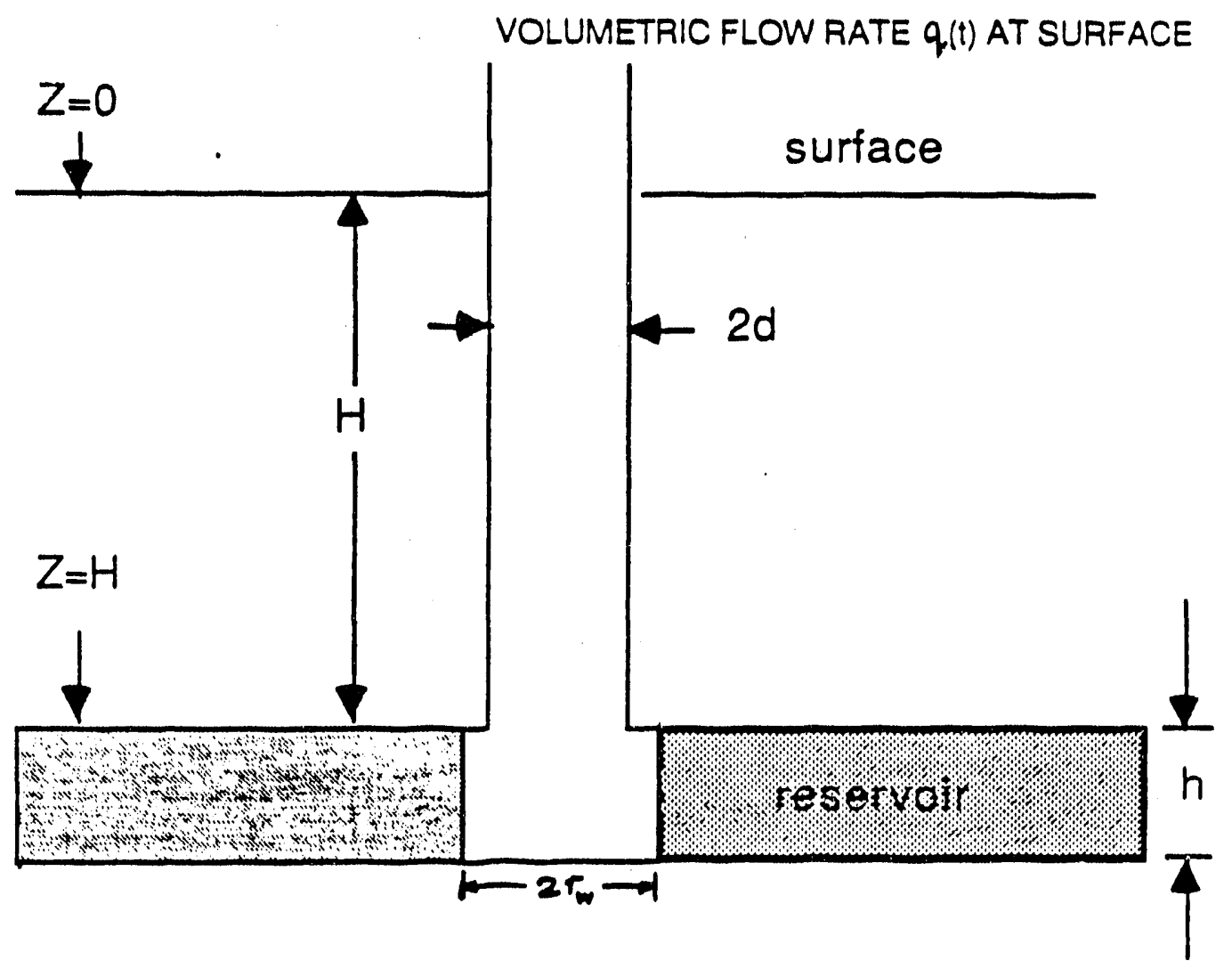

Fig. 5.1 Schematic diagram of well and reservoir 
We will assume $c$ to be constant. Substitution of Eqs. 5.2 and 5.3 in 5.1 yields the the Continuity Equation we will use for this study:

$$
c \frac{\partial p}{\partial t}+c u \frac{\partial p}{\partial z}+\frac{\partial u}{\partial z}=0
$$

That is we will consider velocity in the z-direction in the wellbore only. The other equation we will use for the wellbore is the Navier Stokes Equation. The full form is given in Appendix A. The equation for the wellbore in the $z$ direction is given by:

$$
\begin{gathered}
\rho \frac{D u}{D t}=-\frac{\partial p}{\partial z}+F_{z}+\mu\left(\frac{\partial^{2} u}{\partial x^{2}}+\frac{\partial^{2} u}{\partial y^{2}}+\frac{\partial^{2} u}{\partial z^{2}}\right)+ \\
\left(\xi+\frac{1}{3} \mu\right) \frac{\partial}{\partial x}\left(\frac{\partial w}{\partial x}+\frac{\partial v}{\partial y}+\frac{\partial u}{\partial z}\right)
\end{gathered}
$$

The body force density $F_{z}$ is $-\rho g$ in the weilbore. Expanding Eq. 5.5 and simplifying (see Appendix A for notation):

$$
\rho\left(\frac{\partial u}{\partial t}+u \frac{\partial u}{\partial z}\right)=-\frac{\partial p}{\partial z}-\rho g+\frac{4}{3} \mu \frac{\partial^{2} u}{\partial z^{2}}
$$

Since the second and the fifth term are negligible for the model under consideration the final form of the Navier Stokes Equation is:

$$
\rho \frac{\partial u}{\partial t}+\frac{\partial p}{\partial z}=-\rho g
$$

A similar exercise for the Continuity Equation 5.4 yielded the following dimensional form of the equation:

$$
c \frac{\partial p}{\partial t}+\frac{\partial u}{\partial z}=0
$$

To obtain the equation representing liquid flow in the wellbore we combine Eqs.5.7 and 5.8. Taking the partial derivative of Eq. 5.7 with respect to $t$ :

$$
\rho \frac{\partial^{2} u}{\partial t^{2}}+\frac{\partial^{2} p}{\partial z \partial t}=0
$$


Taking the partial derivative of Eq. 5.8 respect to $z$ :

$$
\frac{\partial^{2} p}{\partial z \partial t}+\frac{1}{c} \frac{\partial^{2} u}{\partial z^{2}}=0
$$

Subtracting Eqs. 5.9 and 5.10:

$$
\frac{\partial^{2} u}{\partial t^{2}}-\frac{1}{\rho c} \frac{\partial^{2} u}{\partial z^{2}}=0
$$

From fluid mechanics [19][21] $c_{0}^{2}=\frac{1}{p c}$ where $c_{0}$ is the wave velocity in the liquid. This subtitution yields the wave equation for the wellbore. For the time period of interest (typical value was 70 seconds, the time period of the observed oscillations), no inertial terms appear in the wellbore equation. The wave equation is:

$$
\frac{\partial^{2} u}{\partial t^{2}}-c^{2} \cdot \frac{\partial^{2} u}{\partial z^{2}}=0
$$

The next step is to couple the wave equation with the diffusivity equation, the complete form of which is:

$$
\frac{1}{r} \frac{\partial p}{\partial r}+\frac{\partial^{2} p}{\partial r^{2}}+c\left(\frac{\partial p}{\partial r}\right)^{2}=\frac{\phi \mu c_{t}}{k} \frac{\partial p}{\partial t}
$$

Ignoring the gradient squared term which is often taken to be negligible, we obtain:

$$
\frac{1}{r} \frac{\partial p}{\partial r}+\frac{\partial^{2} p}{\partial r^{2}}=\frac{\phi \mu c_{t}}{k} \frac{\partial p}{\partial t}
$$


We are thus left with the following governing equations for our model in Fig. 5.1. The wave equation:

$$
\frac{\partial^{2} u}{\partial t^{2}}-c_{s}^{2} \frac{\partial^{2} u}{\partial z^{2}}=0
$$

The diffusivity equation is:

$$
\frac{\partial \Delta p}{\partial t}-\frac{k}{\phi \mu c_{t}} \frac{1}{r} \frac{\partial}{\partial r}\left(r \frac{\partial \Delta p}{\partial r}\right)=0
$$

We replace $p$ by $\Delta p$ since we are concerned only with the change in pressure. The coupling conditions are as follows. The rate of flow through the sand face must equal the rate of liquid storage in the wellbore:

$$
\left.\pi d^{2} u\right|_{x=H}=-\left.\frac{2 \pi r_{w} h k}{\mu} \frac{\partial \Delta p}{\partial r}\right|_{r=r_{w}}
$$

The rate of change of pressure in the wellbore liquid results in a change in vertical velocity in the wellbore:

$$
-\left.c_{\lrcorner}^{2} \rho \frac{\partial u}{\partial z}\right|_{z=H}=\left.\frac{\partial \Delta p}{\partial t}\right|_{r=r_{\omega}}
$$

The boundary and initial conditions are :

$$
\begin{aligned}
u(z=0) & =\frac{q(t)}{\pi d^{2}} \\
\Delta p & \rightarrow 0 \text { as } r \rightarrow \infty
\end{aligned}
$$

To solve this system of equations we introduce Laplace transforms(LT) using the notation:

$$
L\{u\}=\hat{u}=\int_{0}^{\infty} e^{-s t} u(t) d t
$$

Applying LT to Eq. 5.15:

$$
s^{2} \hat{u}-c^{2} \cdot \frac{d^{2} \hat{u}}{d z^{2}}=0
$$

and applying $\mathrm{LT}$ to Eq. 5.16: 


$$
s \Delta \hat{p}-\left(\frac{k}{\phi \mu c_{t} r}\right) \frac{\dot{d}}{d r}\left(r \frac{d \Delta \hat{p}}{d r}\right)=0
$$

In both cases, the initial condition terms are zero. The coupling conditions become:

$$
\begin{aligned}
\left.\pi d^{2} \hat{u}\right|_{z=H} & =-\left.\frac{2 \pi r_{w} h k}{\mu} \frac{\partial \Delta \hat{p}}{\partial r}\right|_{r=r_{w}} \\
-\left.c_{s}^{2} \rho \frac{\partial \hat{u}}{\partial z}\right|_{z=H} & =s \hat{p} \mid r=r_{w} \\
\hat{u}(z=0) & =\frac{\hat{q}(s)}{\pi d^{2}} \\
\Delta \hat{p} & \rightarrow 0 \text { as } r \rightarrow \infty
\end{aligned}
$$

Solving the wave Eq. 5.21:

$$
\hat{u}(z, s)=\frac{\hat{q}(s)}{\pi d^{2}}\left[\operatorname{Cosh}\left(\frac{s}{c_{s}} z\right)+\alpha \operatorname{Sinh}\left(\frac{s}{c_{s}} z\right)\right]
$$

where $\alpha$ is a constant to be determined. Solution of the diffusivity equation 5.22 yields:

$$
\Delta \hat{p}(r, s)=-S \hat{q}(s) \frac{K_{0}\left(\sqrt{\frac{\phi \mu c_{1} s}{k}} r\right)}{\sqrt{\frac{\phi \mu c_{t} r_{w s}^{2}}{k}} K_{1}\left(\sqrt{\frac{\phi \mu c_{t s} s}{k}} r_{w}\right)}\left(\frac{\mu}{2 \pi k h}\right)
$$

where $S$ is a constant to be determined. The coupling equations 5.23-5.26 require:

$$
\pi d^{2}\left(\frac{\hat{q}(s)}{\pi d^{2}}\right)\left[\operatorname{Cosh}\left(\frac{s}{c_{s}} H\right)+\alpha \operatorname{Sinh}\left(\frac{s}{c_{s}} H\right)\right]=-\frac{2 \pi r_{w} h k}{\mu} S \hat{q}(s) \frac{1}{r_{w}}\left(\frac{\mu}{2 \pi k h}\right)
$$

That is:

$$
\operatorname{Cosh}\left(\frac{s}{c_{s}} z\right)-\alpha \operatorname{Sinh}\left(\frac{s}{c_{s}} z\right)=-S
$$

Substituting this condition in Eq. 5.18 yields:

$$
\begin{aligned}
-c_{s}^{2} \rho \frac{\hat{q}(s)}{\pi d^{2}} \frac{s}{c_{s}}\left[\operatorname{Sinh}\left(\frac{s}{c_{s}} z\right)+\alpha \operatorname{Cosh}\left(\frac{s}{c_{s}} z\right)=\right. & s S \hat{q}(s) \\
& \frac{K_{0}\left(\sqrt{\frac{\phi \mu c_{t} r_{w}^{2} s}{k}}\right)}{\sqrt{\frac{\phi \mu c_{t} r_{w}^{2} s}{k}} K_{1}\left(\sqrt{\frac{\phi \mu c_{1} r_{w}^{2} s}{k}}\right)}\left(\frac{\mu}{2 \pi k h}\right)
\end{aligned}
$$


That is:

$$
\operatorname{Sinh}\left(\frac{s}{c_{s}} z\right)+\alpha \operatorname{Cosh}\left(\frac{s}{c_{s}} z\right)=\left(\frac{\mu / 2 \pi k h}{\rho c_{s} / \pi d^{2}}\right) \frac{K_{0}\left(\sqrt{\frac{\phi \mu c_{1} r_{w \rho}^{2}}{k}}\right)}{\sqrt{\frac{\phi \mu c_{1} r_{w}^{2} s}{k}} K_{1}\left(\sqrt{\frac{\phi \mu c_{1} r_{w}^{2} s}{k}}\right)} S
$$

The following linear system results for $\alpha$ and $S$ :

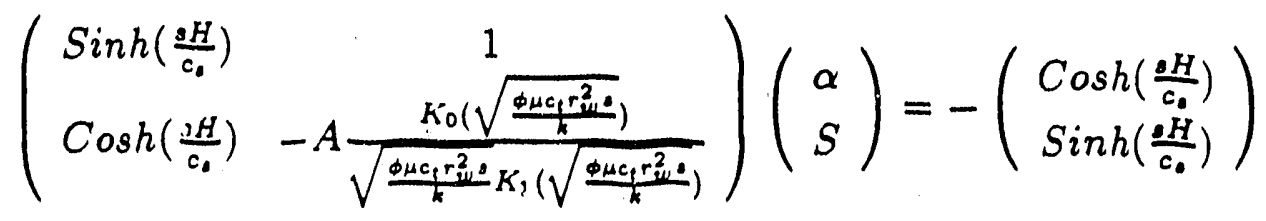

where $A=\frac{\mu / 2 \pi k h}{\rho_{0} / \pi d^{2}}$

To obtain the value of $\alpha$ and $S$ we invert the matrix which yields :

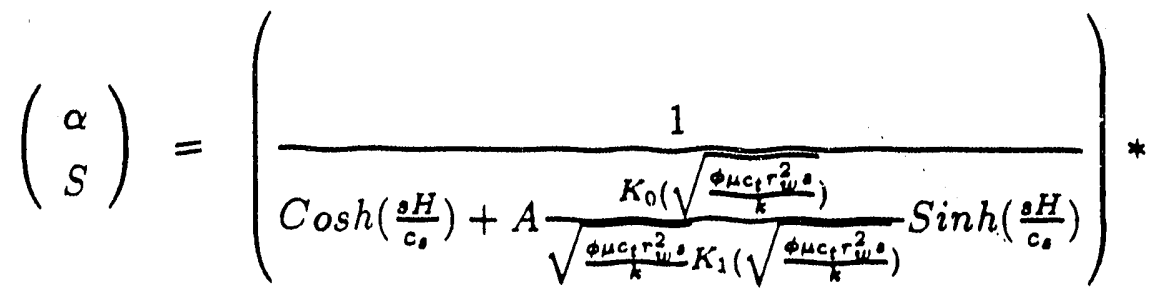

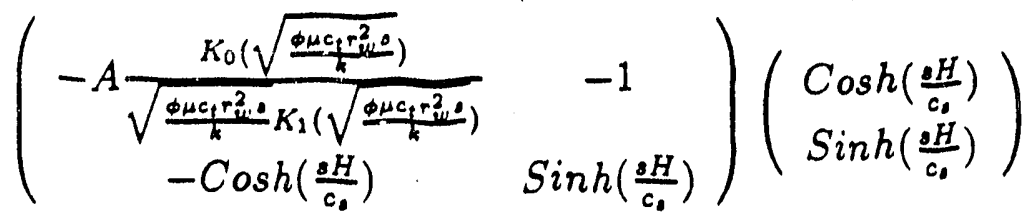

The change in bottom hole pressure is:

$$
\Delta \hat{p}\left(r_{w}, s\right)=-S \hat{q}(s)\left(\frac{\mu}{2 \pi k h}\right) \frac{K_{0}\left(\sqrt{\frac{\phi \mu c_{1} r_{w}^{2} s}{k}}\right)}{\sqrt{\frac{\phi \mu c_{1} r_{w}^{2} s}{k}} K_{1}\left(\sqrt{\frac{\phi c_{0} r_{w i}^{2}}{k}}\right)}
$$

Replacing the value of $S$ by means of Eq. 5.34 yields:

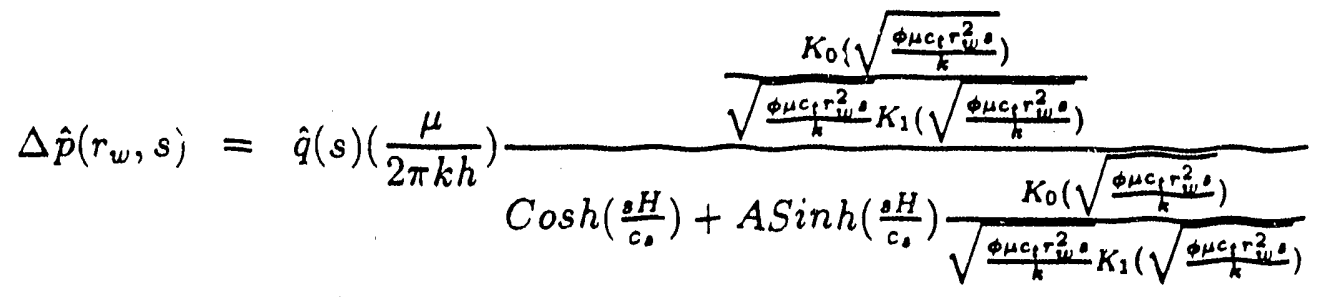

$$
\begin{aligned}
& \text { where } A=\frac{\mu / 2 \pi k h}{\rho c_{s} / \pi d^{2}}
\end{aligned}
$$


This is the complete solution for $\Delta p$ in laplace space which may be inverted to provide the pressure response in time for the imposed flow rate $\hat{q}(s)$. We may also look at the short time and long time approximations of the solution by making the following simplifications.

\section{Long time approximation}

As $s \rightarrow 0:$

$$
\cosh \left(\frac{s H}{c_{s}}\right) \rightarrow 1
$$

and

$$
\sinh \left(\frac{s H}{c_{s}}\right) \rightarrow \frac{s H}{c_{0}}
$$

The expression for $\Delta \hat{p}$ simplifies to:

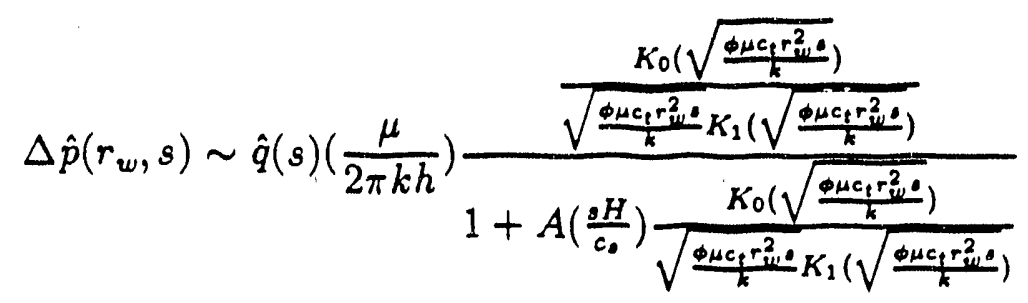

Short time approximation

As $s \rightarrow \infty$ :

$$
\begin{aligned}
\frac{K_{0}\left(\sqrt{\frac{\phi \mu c_{1} \tau_{2 \varepsilon}^{2}}{k}}\right)}{\sqrt{\frac{\phi \mu c_{t} \tau_{w}^{2} s}{k}} K_{1}\left(\sqrt{\frac{\phi \mu c_{t} r_{\mu}^{2} s}{k}}\right)} & \rightarrow 0 \\
\operatorname{Cosh}\left(\frac{s H}{c_{s}}\right) & \sim \operatorname{Sinh}\left(\frac{s H}{c_{s}}\right) \sim e^{s}
\end{aligned}
$$

This leads to the following approximation for $\Delta \hat{p}$ :

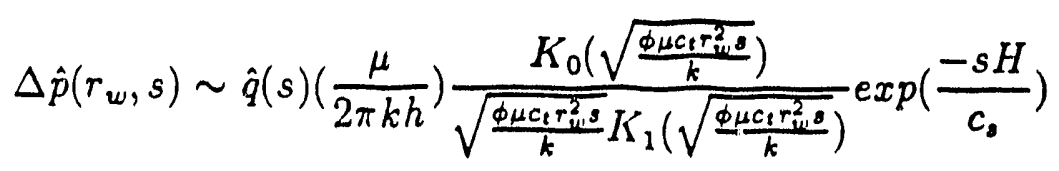


which shows that the downhole pressure response is delayed by the acoustic travel time in the borehole.

The solution can be inverted numerically to study the pressure response when different surface flow rates are imposed on the system.

The flow rate imposed on the model in laplace space was:

$$
\hat{q}(s)=\frac{5,000[1-\exp (-1000 s)]}{s}
$$

This represents a well flowing at $5000 \mathrm{~b} / \mathrm{d}$ for 1000 seconds and then shut in.

To perform the inversion a routine based on the Crurnp [5] method with some modifications was used. This allows excellent inversion characteristics compared to the Stehfest algorithm. The Crump inversion is done in complex space and is computationally more intensive than the Stehfest method. The results of the numerical inversion for the pressure drop vs. time are shown in Fig. 5.2. The results show the presence of oscillations in the system.

\subsection{Results}

A study of the results revealed the following features of the solution:

1. The pressure response shows oscillations with a time period of approximately one second. This is much shorter than the observed time period of 70 seconds for the Mexican field data. The time period may be found to be $\frac{2 L}{c_{0}}$, the time required for the pressure pulse to travel twice the length of the wellbore.

2. After shut in, a pressure rise takes place with a time delay which is equal to the time taken for the pressure pulse to travel the length of the wellbore. The delay time $t_{D}$ is thus $\frac{L}{i_{0}}$. For the plot in Fig. 5.2, a wellbore of length 970 meters and a wave velocity $c_{\text {s }}$ of 1500 meters/sec was used. This yields a delay time of about 0.646 seconds which is approximately verified by Fig. 5.2 .

This result is as one would expect. It has been shown by Holzbausen[14][13] that oscillations due to wellbore inertial effects have a time period of a few seconds. Since 
Fig. 5.2 Pressure drop vs. time for a constant flow rate of $5.000 \mathrm{~b} / \mathrm{d}$ for 1000 seconds and then shut in

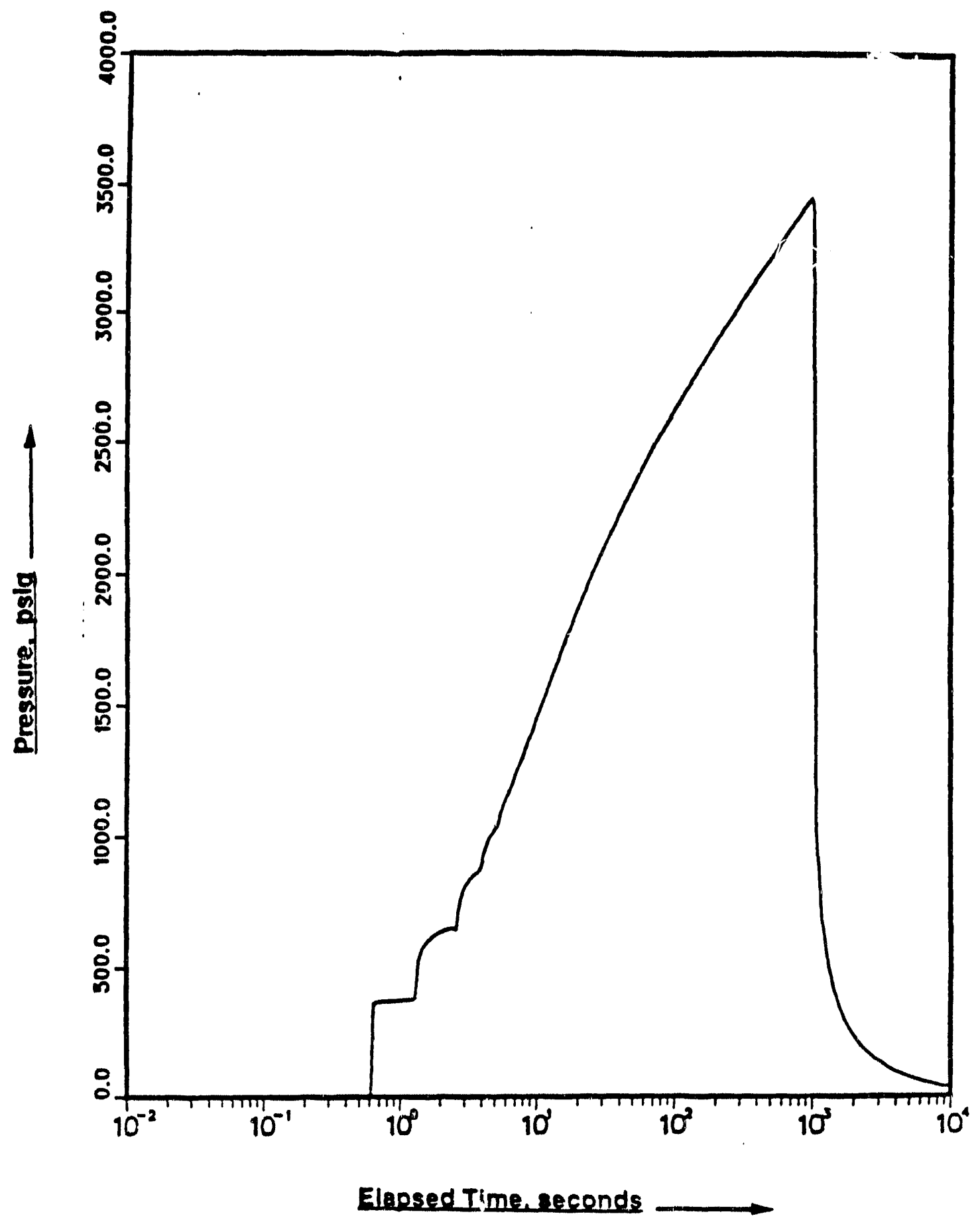


the typical time period of such oscillations is $\frac{2 H}{c_{0}}$, it would take an extremely long pipe length to reduce the frequency of the oscillations to the value seen in the Mexican well. Oise way to increase the time period would be a reduction in the wave velocity $c_{s}$. This would seem to be possible for wave travel through thin apertures(eg. fractures) where the velocity would be reduced due to viscous effects. Due to the large internal diameter of the wellbore and consequently negligible viscous effects, we should not expect to see this effect in a wellbore.

There are other possible sources for wellbore oscillations. An example is Earth tide effects caused by the moon, which can cause oscillations with time period of roughly 12 hours. This is greatly different from the present case in which the time period is much lower ( 70 seconds). Results of calculations for the preceding case lead us to conclude that the cause of the oscillations in the observed field data is not inertial effects in the wellbore. A more likely cause is inertial effects in the formation fractures.

\subsection{Effect of Transducer Time Constant on the Oscillations}

Because oscillations from inertial effects in the weilbore are of a high frequency i.e of the order of one second, it is important to consider the response time of the traisducer used to measure the pressure response. This is of interest because the time constant of most transducers is of the order of one second and this could mask pressure oscillations.

To study this effect, the computed pressure response was mathematically adjusted to obtain the pressure response a transducer would record. This was done by dividing the pressure response in laplace space by a first order time constant for the transducer. Thus if $\Gamma$ is the time constant for the transducer:

$$
p_{\text {recorded }}=\frac{p_{\text {true }}}{1+\Gamma}
$$

Calculations were made for different time constants, and results for two guage time constants ( 0.1 and 0.6 seconds) are shown in Figs. 5.3 and 5.4. Figure 5.3 shows 
the model pressure response as a dotted line, and the solid line represents the pressure response a pressure transducer would measure. The latter incorporates the effect of the transducer time constant of 0.1 second. There is a small difference between the two curves.

However as the time constant $\Gamma$ is increased to $) .6$ second (see Fig. 5.4) the pressure oscillations are nearly masked by the poor response of the transducer. This has practical implications. If we wish to measure such high frequency oscillations, we should choose a transducer carefully. It can also mean that inertial pressure oscillations may exist but not be measured by unresponsive pressure devices.

The next section presents results computed for linear flow to a well with inertial effects in a fracture. 
Fig. 5.3. Pressure drop vs. time for model and a transducer with a 0.1 second time constant

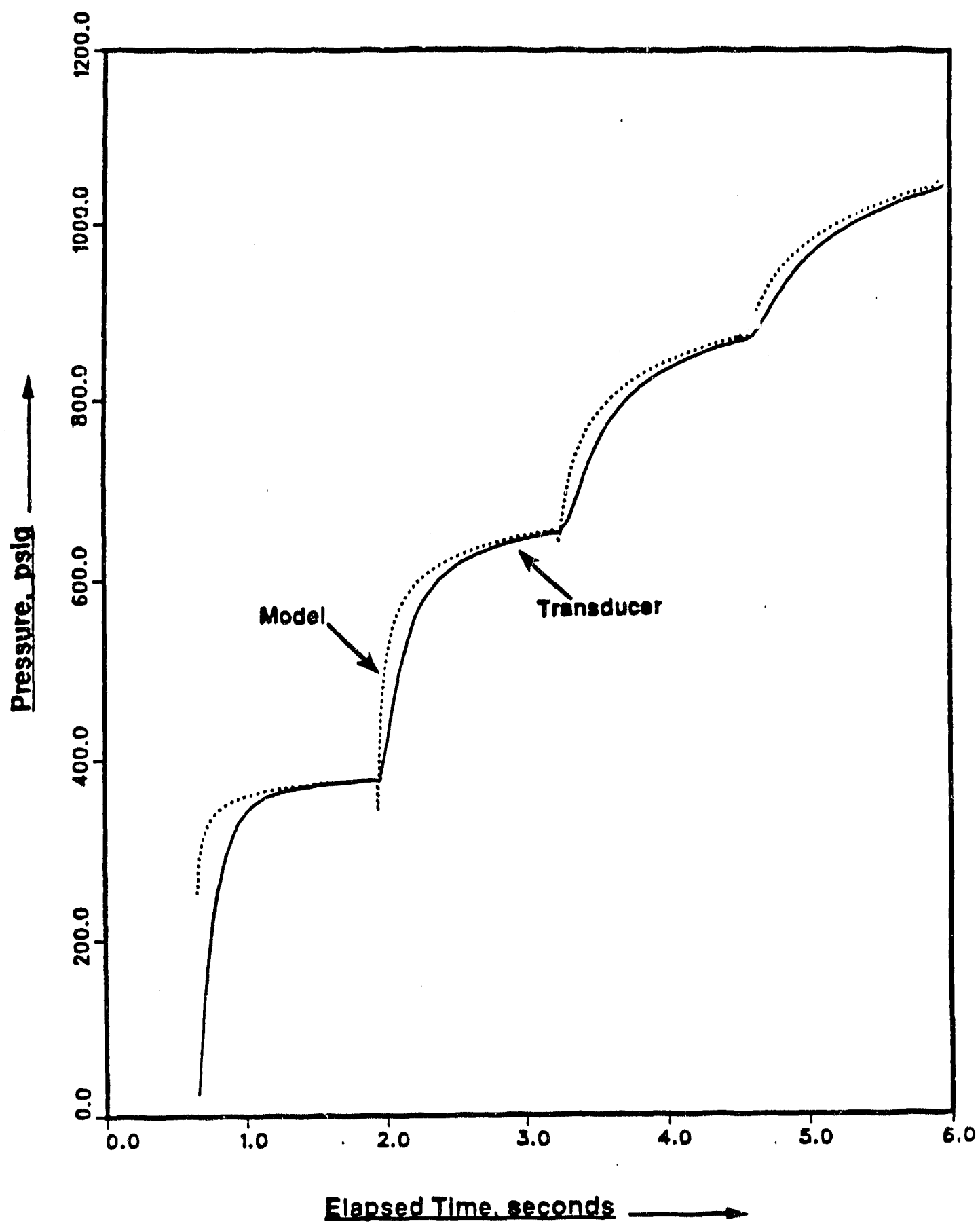


Fig. 5.4. Pressure drop vs. time for model and a transducer with a 0.6 second time constant

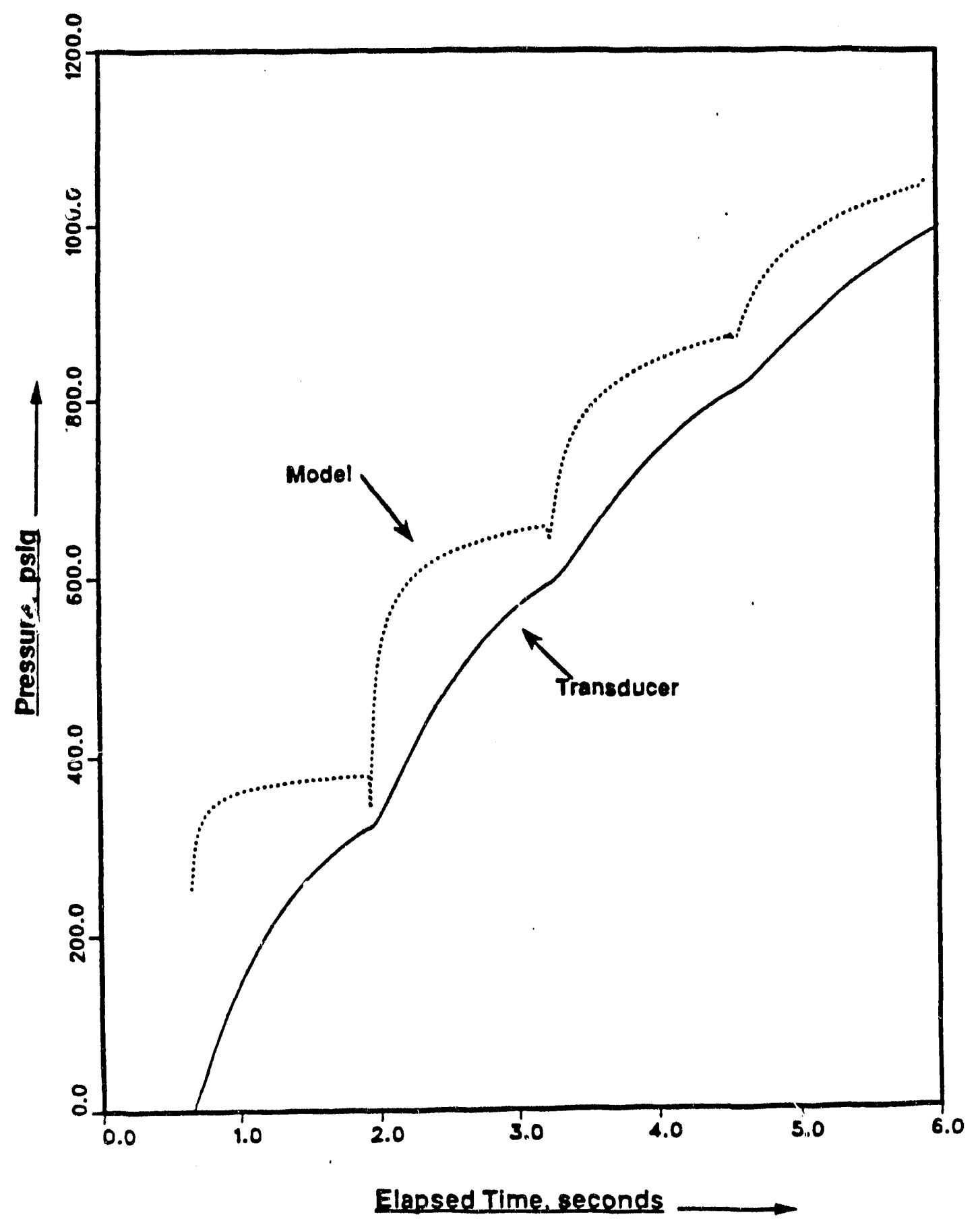




\section{Section 6}

\section{Cartesian Solution}

The next model considers a linear flow to a well with inertial effects in a single fracture. We thus assume:

- No inertial effects in the wellbore,

- A penny-shaped fracture communicating with the wellbore, and

- Instantaneous equalization of pressure between the fracture and the matrix. The matrix has a large storativity.

The model is shown in Figs. 6.1 and 6.2.

\subsection{Continuity Equation}

The continuity equation (in cartesian co-ordinates) for compressible, viscous flow is:

$$
\frac{\partial \rho}{\partial t}+\frac{\partial}{\partial x}(\rho u)+\frac{\partial}{\partial y}(\rho v)+\frac{\partial}{\partial z}(\rho w)=0
$$

where $u, v$ and $w$ are velocities in the $x, y$ and $z$ directions respectively. For a simple one dimensional flow:

$$
\begin{aligned}
v & =0 \\
w & =0
\end{aligned}
$$




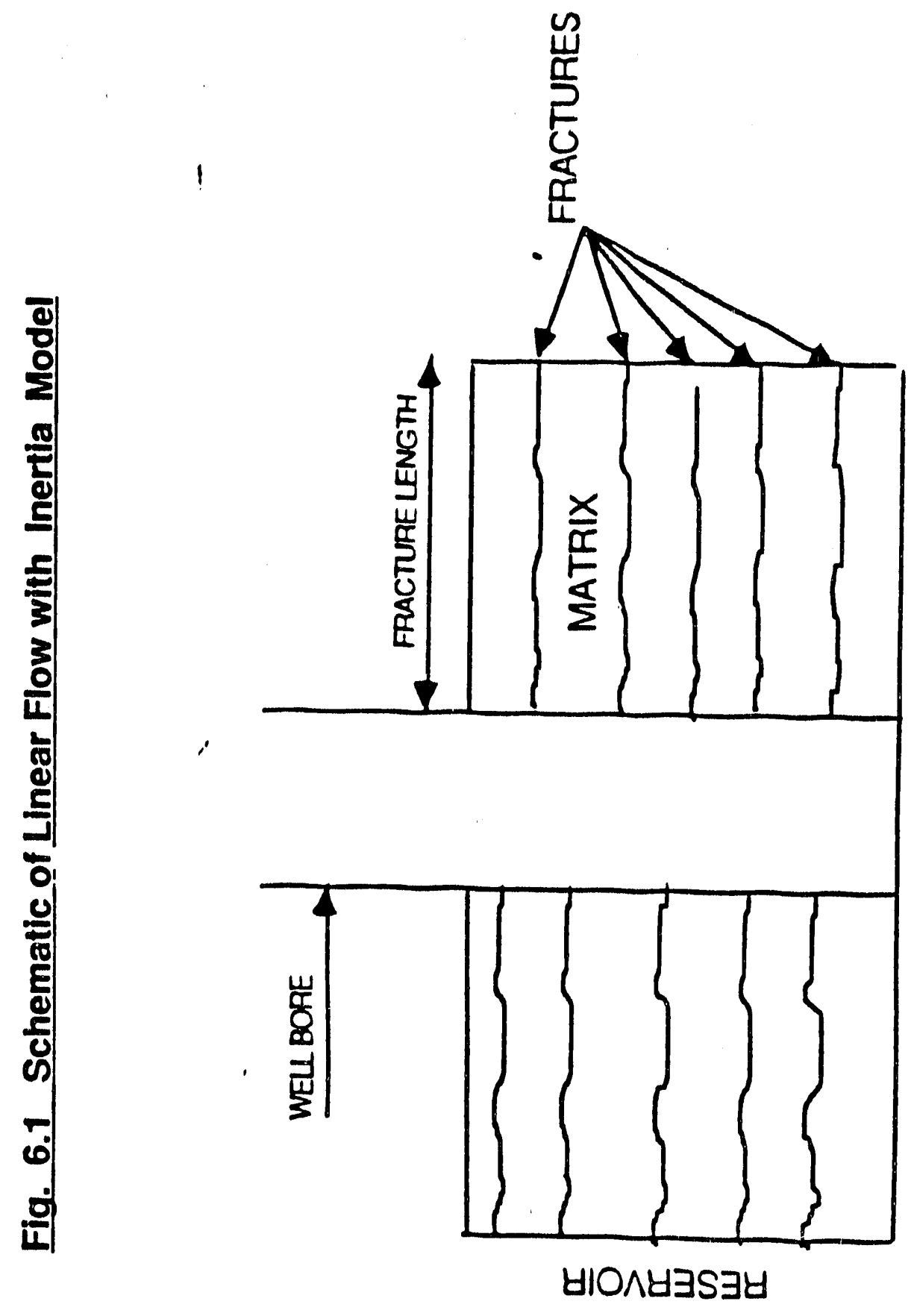




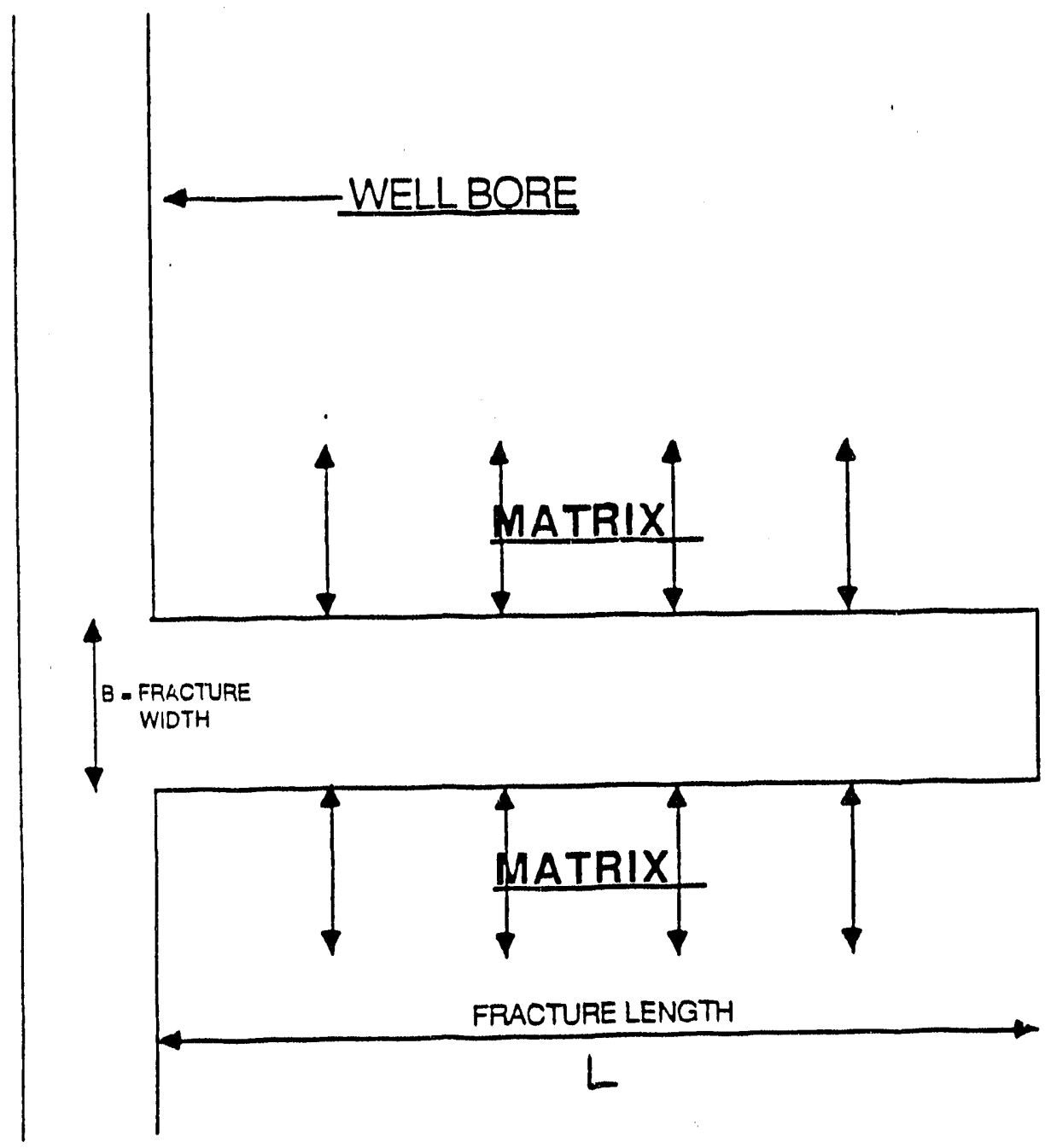

Fig. 6.2. Schematic of a single fracture in the Linear Flow with Inertia Model 
From Eqs. 6.1 and 6.2:

$$
\frac{\partial \rho}{\partial t}+\frac{\partial}{\partial x}(\rho u)=0
$$

Substituting the relationship between pressure $p$ and density $\rho$ for a liquid with constant compressibility i.e. $d \rho=\rho c d p$ and for $c_{s}$ :

$$
\frac{1}{c_{s}^{2}} \frac{\partial p}{\partial t}+\rho \frac{\partial u}{\partial x}+u \frac{1}{c_{s}^{2}} \frac{\partial p}{\partial x}=0
$$

Let the storativity ratio $\Omega$ be the ratio of the reservoir pore volume to the fracture

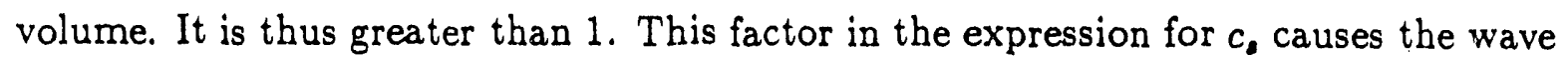
velocity to decrease in a fracture. Normally the wave vela-ity is given by $c_{s}=\frac{1}{\sqrt{p c}}$, but in this case it is given by $c_{s}=\frac{1}{\sqrt{p n c}}$. Dropping the third term in Eq. 6.4 since it is insignificant for the model under consideration we obtain the final form of the Continuity Equation as:

$$
\Omega c \frac{\partial p}{\partial t}=-\frac{\partial u}{\partial x}
$$

We consider the Navier Stokes equations in the next section.

\subsection{Navier Stokes Equation}

The complete system of Navier Stokes Equations (in Cartesian Co-ordinates) are given in the appendix. We will consider only the equation in the $x$ direction. This equation is:

$$
\begin{gathered}
\rho \frac{D u}{D t}=-\frac{\partial p}{\partial x}+F_{x}+\mu\left(\frac{\partial^{2} u}{\partial x^{2}}+\frac{\partial^{2} u}{\partial y^{2}}+\frac{\partial^{2} u}{\partial z^{2}}\right)+ \\
\left(\xi+\frac{1}{3} \mu\right) \frac{\partial}{\partial x}\left(\frac{\partial u}{\partial x}+\frac{\partial v}{\partial y}+\frac{\partial w}{\partial z}\right)
\end{gathered}
$$

This equation can be simplified since $v$ and $w$ are negligible, and $u$ varies only in the $x$ and $z$ directions. 


$$
\rho\left(\frac{\partial u}{\partial t}+u \frac{\partial u}{\partial x}\right)=-\frac{\partial p}{\partial x}+\mu\left(\frac{\partial^{2} u}{\partial x^{2}}+\frac{\partial^{2} u}{\partial z^{2}}\right)
$$

Dropping insignificant terms (two and four) in Eq. 6.7 we obtain the final form of the Navier Stokes equation" as:

$$
\frac{\partial u}{\partial t}=-\frac{\partial p}{\partial x}+\mu \frac{\partial^{2} u}{\partial z^{2}}
$$

The next step is to solve Eqs. 6.5 and 6.8 simultaneously.

\subsection{Solution of System Equations}

1. The Navier Stokes Equation is:

$$
\rho \frac{\partial u}{\partial t}=-\frac{\partial p}{\partial x}+\mu \frac{\partial^{2} u}{\partial z^{2}}
$$

2. The Continuity Equation is:

$$
\Omega c \frac{\partial p}{\partial t}=-\frac{\partial u}{\partial x}
$$

We make the following assumptions:

(a) We assume a velocity profile for fluid flow between parallel plates, and

(b) We average both equations in the $z$ direction since we assume that there is no flow in that direction.

The first assumption permits the use of the following equation for fluid flow between parallel plates.

$$
\begin{aligned}
u(z) & =\frac{6 z}{b}\left(1-\frac{z}{b}\right) u \\
\frac{\partial u}{\partial z} & =\left(\frac{6}{b}-\frac{12 z}{b^{2}}\right) u
\end{aligned}
$$

The second assumption allows us to average the two equations along the $z$ direction. Using the notation that the averaged variables are denoted by a bar 
above the variable, i.e., $\bar{u}$ is the averaged value of $u$, the Navier Stokes Eq. 6.9 becomes:

$$
\rho \frac{\partial \bar{u}}{\partial t}=-\frac{\partial \bar{p}}{\partial x}+\frac{\mu}{b}\left(\left.\frac{\partial \bar{u}}{\partial z}\right|_{z=b}-\left.\frac{\partial \bar{u}}{\partial z}\right|_{z=0}\right)
$$

or:

$$
\begin{aligned}
\rho \frac{\partial \bar{u}}{\partial t} & =-\frac{\partial \bar{p}}{\partial x}+\frac{\mu}{b}\left(-\frac{6}{b}-\frac{6}{b}\right) \bar{u} \\
\rho \frac{\partial \bar{u}}{\partial t} & =-\frac{\partial \bar{p}}{\partial x}-\frac{12 \mu}{b^{2}} \bar{u}
\end{aligned}
$$

Assuming that the liquid is slightly compressible and differentiating with respect to $t$ :

$$
\rho \frac{\partial^{2} \bar{u}}{\partial t^{2}}=-\frac{\partial^{2} \bar{p}}{\partial x \partial t}-\frac{12 \mu}{b^{2}} \frac{\partial \bar{u}}{\partial t}
$$

Similarly averaging the Continuity equation 6.10 :

$$
\Omega c \frac{\partial \bar{p}}{\partial t}=-\frac{\partial \bar{u}}{\partial x}
$$

Differentiating with respect to $x$ :

$$
-\operatorname{sic} \frac{\partial^{2} \bar{p}}{\partial x \partial t}=\frac{\partial^{2} \bar{u}}{\partial x^{2}}
$$

Combining the Continuity and Navier Stokes equations by substituting the value of the cross derivative from Eq. 6.16 into Eq. 6.14:

$$
\frac{\partial^{2} \bar{u}}{\partial t^{2}}-\frac{1}{\rho \Omega c} \frac{\partial^{2} \bar{u}}{\partial x^{2}}+\frac{12 \mu}{\rho b^{2}} \frac{\partial \bar{u}}{\partial t}=0
$$


We convert this equation into a non-dimensional form so that it will be easier to manipulate. The following dimensionless groups will be substituted in both the Continuity and the Navier Stokes equations.

$$
\begin{aligned}
p_{D} & =\frac{b^{2} \Delta p}{12 \mu U L} \\
u_{D} & =\frac{u}{U} \\
x_{D} & =\frac{x}{L} \\
t_{D} & =\frac{t}{(L \sqrt{\rho \Omega c})}=\frac{c_{D}}{L} t
\end{aligned}
$$

Also let:

$$
\beta=\frac{12 \mu L}{\rho b^{2} c_{d}}
$$

Using Eqs. 6.18-6.22:

$$
\frac{\partial^{2} \bar{u}_{D}}{\partial t_{D}{ }^{2}}+\beta \frac{\partial \bar{u}_{D}}{\partial t_{D}}=\frac{\partial^{2} \bar{u}_{D}}{\partial x_{D}^{2}}
$$

Taking the Laplace Transform of Eq. 6.23 and remembering that the zero time value of $\hat{u_{D}}$ is zero:

$$
\frac{\partial^{2} \hat{u_{D}}}{\partial x^{2}}-\hat{u_{D}}\left(s^{2}+\beta s\right)=0
$$

Let $\Lambda=s^{2}+\beta s$. Then:

$$
\frac{\partial^{2} \hat{u_{D}}}{\partial x^{2}}-\Lambda \hat{u_{D}}=0
$$

The solution of Eq. 6.25 is: 


$$
\hat{u_{D}}(x, s)=C_{1} \operatorname{Sinh}(\Lambda x)+C_{2} \operatorname{Cosh}(\Lambda x)
$$

In order to obtain the full solution we must determine the two constants $C_{1}$ and $C_{2}$, and invert the resulting expression to real space.

The Boundary conditions that govern the solution is:

$$
\begin{array}{r}
\hat{u_{D}}=U(s), \text { for } x_{D}=0 \\
\hat{u_{D}}=0, \text { for } x_{D}=1 \\
\dot{u_{D}}=0 \text { and } \hat{u_{D}}=0, \text { for } x, t=0 \\
\hat{p_{D}}=0 \text { for } x, t=0
\end{array}
$$

where $U(s)$ is a specified value for the velocity (in laplace space) at the mouth of the fracture where the fracture meets the wellbore. Solving Eq. 6.26 for the given boundary conditions yields:

$$
\hat{u_{D}}(x, s)=-[\operatorname{Coth}(\Lambda) \operatorname{Sinh}(\Lambda x)+\operatorname{Cosh}(\Lambda x)] U(s)
$$

because:

$$
\begin{aligned}
& C_{1}=-\operatorname{Coth}(\Lambda) \\
& C_{2}=1
\end{aligned}
$$

Having determined $C_{1}$ and $C_{2}$, we have determined the velocity of the fluid flow in the fracture, but bave yet to determine the pressure response. To determine the pressure response in laplace space we use the continuity Eq. 6.15 which relates velocity and pressure:

$$
\Omega c \frac{\partial \bar{p}}{\partial t}=-\frac{\partial \bar{u}}{\partial x}
$$


or in dimensionless form:

$$
\beta \frac{\partial \overline{p_{D}}}{\partial t_{D}}=-\frac{\partial \overline{u_{D}}}{\partial x_{D}}
$$

Taking the Laplace transform of Eq. 6.31 and remembering that the zero time value of the real space pressure drop is zero:

$$
s \beta \hat{p_{D}}=-\frac{\partial \hat{u_{D}}}{\partial x_{D}}
$$

or:

$$
\hat{p_{D}}=-\frac{1}{s \beta} \frac{\partial \hat{u_{D}}}{\partial x_{D}}
$$

The solution for the velocity $\hat{u_{D}}$ in Laplace space from Eq. 6.28 is:

$$
\hat{u_{D}}(x, s)=-\operatorname{Coth}(\Lambda) \operatorname{Sinh}(\Lambda x)+\operatorname{Cosh}(\Lambda x)
$$

Eq. 6.34 can be differentiated with respert to $x$ and the result substituted into Eq. 6.33 which relates velocity $\hat{u}_{D}$ to pressure $\hat{P E}$. Thus from Eq. 6.34 :

$$
\frac{\partial \hat{u_{D}}}{\partial x_{D}}=-[\Lambda \operatorname{Coth}(\Lambda)] U(s)
$$

Substituting Eq. 6.34 into Eq. 6.33:

$$
p_{D}(x, s)=-\frac{U(s) \Lambda}{s \beta} \operatorname{Coth}(\Lambda)
$$

This solution maybe evaluated for different values of $\beta$ by numerical inversion. The Crump algorithm in complex space was used for inversion. For the conditions of the Mexican well test given in Table $2.1, \beta$ is approximately unity. For $\Omega \approx 10^{3}-10^{4}$ and $c=4.35 \times 10^{-10} \mathrm{~Pa}^{-1}$, the ratio $\frac{L}{b^{2}}$ is calculated:

$$
\frac{L}{b^{2}}=3.57 \times 10^{6}
$$

This may help to estimate the length of the frackure. Figs. 6.3 and 6.4 present results of the inversion of $E_{q}$. 6.36. 
Fig. 6.3 Dimensionless pressure vs. dimensionless time for beta=1.0 and for a dimensionless production time of 10.000

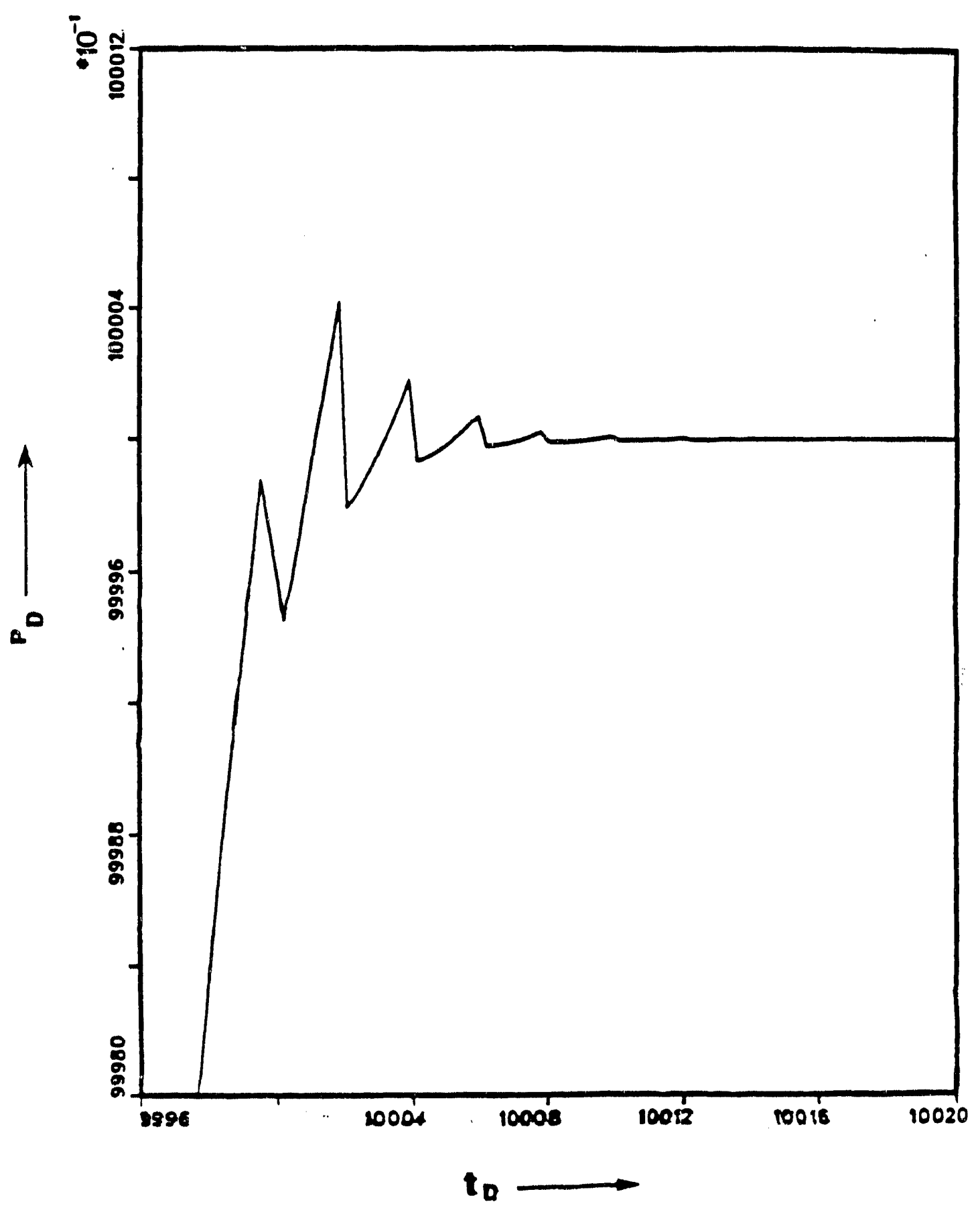


Fig. 6.4 Dimensionless pressure vs. dimensionless time for beta $=1.0$ and for a tranducer time constant of 0.5 seconds

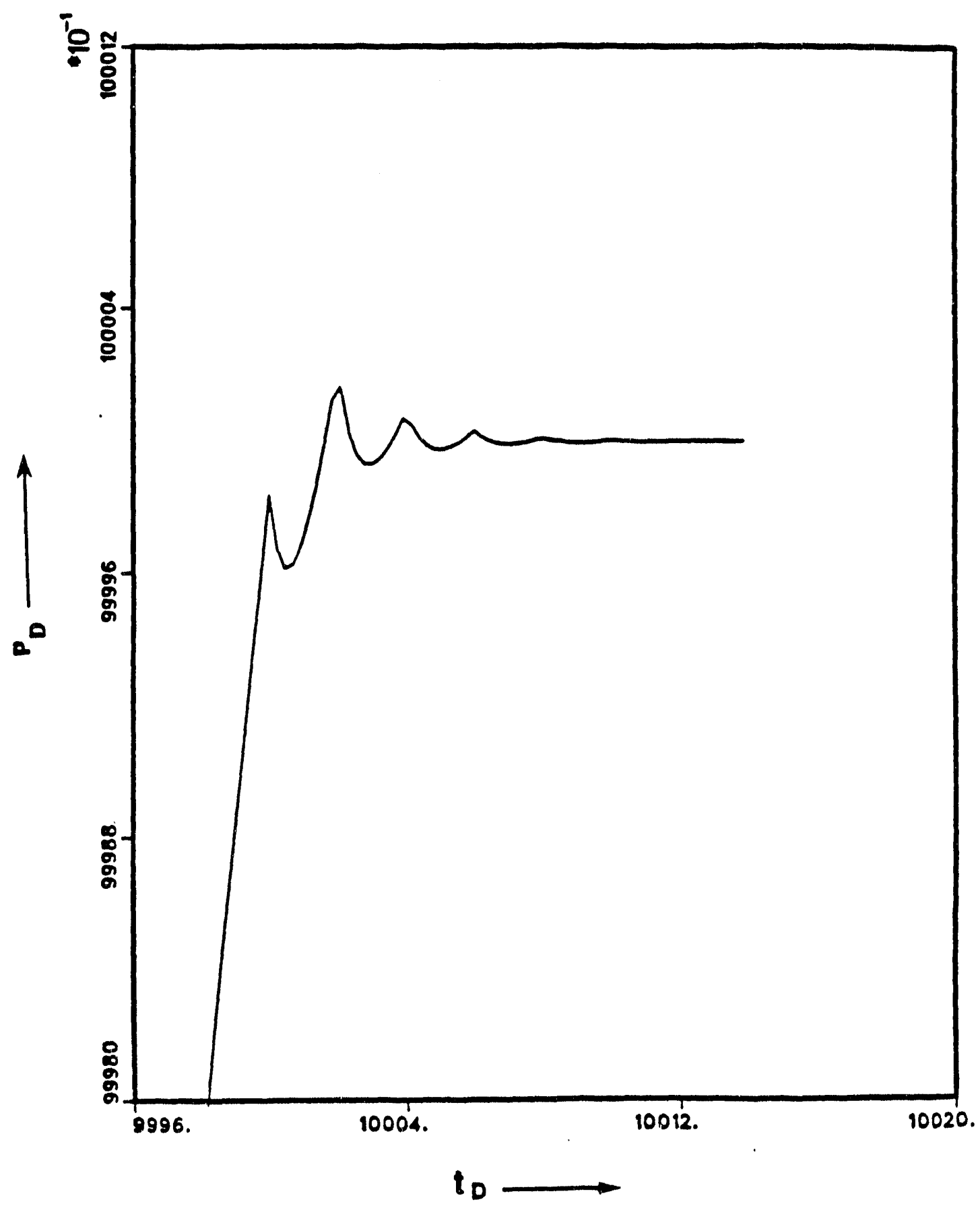




\subsection{Features of the Solution}

From the results shown in Figs. 6.3 and 6.4 we observe:

(a) The pressure oscillations show a time period of approximately two units which for the Mexican well conditions is about 70 seconds.

(b) The oscillations are sharp which may be attributed to the choice of a cartesian formation flow geometry rather than the correct field flow geometry.

(c) Figure 6.4 shows the computed pressure response for a finite time constant of 0.5 seconds. There is little difference between the results on Figs. 6.3 and 6.4 because the oscillations are of a low frequency, and the time constant of the transducer is an order of magnitude smaller than the time period of the computed oscillations.

This result confirms that the main features of the model match the observed pressure response for the field test. Radial formation flow to the well solution is presented in Appendix B. 


\section{Section 7}

\section{Future Work}

The radial solution should be inverted to real space. For this purpose it is necessary to evaluate Bessel functions in complex space. The required Bessel functions are not available for complex arguments in the IMSL library of routines. However a library of functions including these has been obtained from the U.S.Naval Weapons Laboratory. This code is extremely long (more than 2500 lines) and it is now available on the department computers. Details of the library are available in a manual titled "NSWC Library of Mathematics Subroutines ". A copy of this manual may be found in the Petroleum Engineering Department at Stanford.

These functions have been tested and will be used to invert the laplace space solution in complex space. A proprietory algorithm was obtained from the Schlurnberger Doll Research Center (SDR) which improves on the Crump method. The Crump method is able to invert square waves witisut oscillations. This algorithm was written at SDR by Dr. T.S. Ramakrishnan and is available directly from him. This algorithm permits instantaneous shut in conditions in the solution in laplace space without causing spurious oscillations. The Stehfest [18] Laplace transform inversion algorithm does not invert oscillations of a high frequency very well. 
The major conclusions from this work are:

(a) Inertia in the wellbore cannot explain the long time period of the oscillations observed in the Mexican well test data.

(b) Inertial effects in a fracture in the formation may explain the period of oscillations in the field test data. In view of the highly idealized nature of the fracture considered, it may not be the only explanation.

More work should be done on inertia in double porosity media. The work done on the radial flow fracture is of a very idealized type and is presented in Appendix B. 


\section{Section 8}

\section{Symbols}

$$
\begin{array}{ll}
r_{w} & =\text { radius of producing formation } \\
\mathrm{b} & =\text { fracture width } \\
c_{f} & =\text { fracture compressibility } \\
c_{m} & =\text { matrix compressibility } \\
c_{s} & =\text { wave speed in tluid } \\
c_{t} & =\text { total compressibility } \\
\mathrm{f}(\mathrm{s}) & =\text { Laplace space function } \\
\mathrm{g} & =\text { gravitational constant } \\
\mathrm{H} & =\text { length of wellbore } \\
\mathrm{h} & =\text { formation thickness } \\
I_{0}(x) & =\text { modified Bessel function, first kind, zero order } \\
I_{1}(x) & =\text { modified Bessel function, first kind, first order } \\
k & =\text { fracture permeability } \\
K_{0}(x) & =\text { modified Bessel function, second kind, zero order } \\
K_{1}(x) & =\text { modified Bessel function, second kind, first order } \\
\mathrm{L} & =\text { Length of fracture } \\
\mathrm{m} & =\text { slope of linear PDF }
\end{array}
$$




$$
\begin{aligned}
& p_{D_{f}} \quad=\text { dimensionless fracture pressure } \\
& p_{D_{m}} \quad=\text { dimensionless matrix pressure } \\
& p_{D_{w}} \quad=\text { dimensionless well bore pressure } \\
& p \quad=\text { fluid pressure in the wellbore or fracture } \\
& p_{i} \quad=\text { initial reservoir pressure } \\
& p_{m} \quad=\text { matrix fluid pressure } \\
& p_{w f} \quad=\text { wellbore flowing pressure } \\
& \mathrm{q}(\mathrm{t}) \quad=\text { flow rate at wellhead } \\
& r=\text { radial coordinate } \\
& \text { ro }=\text { dimensionless radial coordinate } \\
& r_{w} \quad=\text { wellbore radius } \\
& \mathrm{s} \quad=\text { Laplace parameter } \\
& \mathrm{t} \quad=\text { time } \\
& t_{D} \quad=\text { dimensionless time } \\
& \gamma \quad=1.781 \text {, exponential of Euler's constant } \\
& \lambda=\text { dimensionless interporosity flow coefficient } \\
& \tau=\text { transducer time constant } \\
& \mu \quad=\text { viscosity } \\
& \phi_{f} \quad=\text { fracture porosity } \\
& \phi_{m} \quad=\text { matrix porosity } \\
& \Omega \quad \text { = ratio of reservoir to fracture volume } \\
& \theta \quad=\text { radial coordinate } \\
& U \quad \text { = velocity at the mouth of the fracture at the wellbore } \\
& \rho=\text { density of fluid } \\
& u \quad=\text { velocity in the } \mathrm{x} \text { direction } \\
& v \quad=\text { velocity in the } \mathrm{y} \text { direction } \\
& v_{r} \quad=\text { velocity in the } \mathrm{r} \text { direction } \\
& v_{\theta} \quad=\text { velocity in the } \theta \text { direction } \\
& v_{z} \quad=\text { velocity in the } z \text { direction } \\
& w \quad=\text { velocity in the } z \text { direction } \\
& x=\text { cartesian coordinate }
\end{aligned}
$$




$$
\begin{array}{ll}
y & =\text { cartesian coordinate } \\
z & =\text { cartesian coordinate } \\
\beta & =\text { dimensionless constant } \\
\Lambda & =\text { dimensionless constant defined as } \sqrt{s^{2}+\beta s}
\end{array}
$$




\section{Section 9}

\section{Appendix A}

This appendix summarizes the Navier Stokes Equations and the Continuity Equations for flow in a porous medium.

\subsection{Navier Stokes Equations}

This section gives the full form of the Navier Stokes Equations of Motion for a compressible fluid with constant viscosity in both the cartesian and the radial co-ordinate form.

\subsubsection{Cartesian Co-ordinates}

The following forms of the Navier Stokes equations are sufficiently accurate for most physical problems. Here $u, v$ and $w$ are the velocities in the $x, y$ and the $z$ direction respectively and:

$$
\frac{D}{D t}=\frac{\partial}{\partial t}+u \frac{\partial}{\partial x}+v \frac{\partial}{\partial y}+w \frac{\partial}{\partial z}
$$


The equations are:

$$
\begin{aligned}
\rho \frac{D u}{D t}= & -\frac{\partial p}{\partial x}+F_{x}+\mu\left(\frac{\partial^{2} u}{\partial x^{2}}+\frac{\partial^{2} u}{\partial y^{2}}+\frac{\partial^{2} u}{\partial z^{2}}\right)+ \\
& \left(\xi+\frac{1}{3} \mu\right) \frac{\partial}{\partial x}\left(\frac{\partial u}{\partial x}+\frac{\partial v}{\partial y}+\frac{\partial w}{\partial z}\right) \\
\rho \frac{D v}{D t}= & -\frac{\partial p}{\partial y}+F_{y}+\mu\left(\frac{\partial^{2} v}{\partial x^{2}}+\frac{\partial^{2} v}{\partial y^{2}}+\frac{\partial^{2} v}{\partial z^{2}}\right)+ \\
& \left(\xi+\frac{1}{3} \mu\right) \frac{\partial}{\partial y}\left(\frac{\partial u}{\partial x}+\frac{\partial v}{\partial y}+\frac{\partial w}{\partial z}\right) \\
\rho \frac{D w}{D t}= & -\frac{\partial p}{\partial z}+F_{z}+\mu\left(\frac{\partial^{2} w}{\partial x^{2}}+\frac{\partial^{2} w}{\partial y^{2}}+\frac{\partial^{2} w}{\partial z^{2}}\right)+ \\
& \left(\xi+\frac{1}{3} \mu\right) \frac{\partial}{\partial z}\left(\frac{\partial u}{\partial x}+\frac{\partial v}{\partial y}+\frac{\partial w}{\partial z}\right)
\end{aligned}
$$

\subsubsection{Radial Co-ordinates}

Here $v_{r}, v_{\theta}$ and $v_{z}$ are the fluid velocities in the $r, \theta$ and $z$ directions respectively and:

$$
\begin{aligned}
\frac{D}{D t} & =\frac{\partial}{\partial t}+v_{r} \frac{\partial}{\partial r}+\frac{v_{\theta}}{r} \frac{\partial}{\partial \theta}+v_{z} \frac{\partial}{\partial z} \\
\nabla \cdot \vee & =\frac{1}{r} \frac{\partial}{\partial r}\left(r v_{r}\right)+\frac{1}{r} \frac{\partial v_{\theta}}{\partial \theta}+\frac{\partial v_{z}}{\partial z}
\end{aligned}
$$

and $V$ is the velocity vector. The equations are:

$$
\begin{aligned}
\rho\left[\frac{D v_{r}}{D t}-\frac{v_{\theta}^{2}}{r}\right]= & -\frac{\partial p}{\partial r}+F_{r}+\mu\left[\nabla^{2} v_{r}-\frac{v_{r}}{r^{2}}-\frac{2}{r^{2}} \frac{\partial v_{\theta}}{\partial \theta}\right]+ \\
& {\left[\xi+\frac{1}{3} \mu\right] \frac{\partial}{\partial r}(\nabla \cdot \vee) } \\
\rho\left[\frac{D v_{\theta}}{D t}-\frac{v_{r} v_{\theta}}{r}\right]= & -\frac{1}{r} \frac{\partial p}{\partial \theta}+F_{\theta}+\mu\left[\nabla^{2} v_{\theta}-\frac{v_{\theta}}{r^{2}}+\frac{2}{r^{2}} \frac{\partial v_{r}}{\partial \theta}\right]+ \\
& {\left[\xi+\frac{1}{3} \mu\right] \frac{\partial}{\partial \theta}(\nabla \cdot \vee) }
\end{aligned}
$$




$$
\begin{aligned}
\rho \frac{D v_{z}}{D t}= & -\frac{\partial p}{\partial z}+F_{z}+\mu \nabla^{2} v_{z}+ \\
& {\left[\xi+\frac{1}{3} \mu\right] \frac{\partial}{\partial z}(\nabla \cdot \vee) }
\end{aligned}
$$

\subsection{Continuity Equation}

The continuity equation in the radial and cartesian form is given below. In the cartesian form for a general compressible fluid:

$$
\frac{\partial \rho}{\partial t}+\frac{\partial}{\partial x}(\rho u)+\frac{\partial}{\partial y}(\rho v)+\frac{\partial}{\partial z}(\rho w)=0
$$

In the radial form:

$$
\frac{\partial \rho}{\partial t}+\frac{1}{r} \frac{\partial}{\partial r}\left(r \rho v_{r}\right)+\frac{1}{r} \frac{\partial}{\partial \theta}\left(\rho v_{\theta}\right)+\frac{\partial}{\partial z}\left(\rho v_{z}\right)=0
$$




\section{Section 10}

\section{Appendix B}

We make the following assumptions:

(a) No inertial effects in the wellbore,

(b) A horizontal penny-shaped fracture communicating with the wellbore, and

(c) Instantaneous equalization of pressure between the fracture and the matrix.

\subsection{Continuity Equation}

The Continuity Equation (in radial co-ordinates) for cumpressible, viscous flow is:

$$
\frac{\partial \rho}{\partial t}+\frac{1}{r} \frac{\partial}{\partial r}\left(r \rho v_{r}\right)+\frac{1}{r} \frac{\partial}{\partial \theta}\left(\rho v_{\theta}\right)+\frac{\partial}{\partial z}\left(\rho v_{z}\right)=0
$$

where, $v_{r}, v_{\theta}$ and $v_{z}$ are the fluid velocities in the $r, \theta$ and $z$ directions, respectively.

For the schematic of Fig. 6.1:

$$
v_{q}=0
$$


and

$$
v_{z}=0
$$

Dropping the third and fourth terms in Eq. 10.1 we obtain on expansion:

$$
\frac{\partial \rho}{\partial t}+\frac{1}{r} \rho v_{r}+\rho \frac{\partial v_{r}}{\partial r}+v_{r} \frac{\partial \rho}{\partial r}=0
$$

The isothermal compressibility of a liquid is defined as:

$$
c=\frac{1}{\rho}\left(\frac{\partial \rho}{\partial p}\right)_{T}
$$

Also the wave velocity $c_{s}$ is given by:

$$
c_{s}=\frac{1}{\sqrt{\rho \Omega c}}
$$

Substituting Eqs. 10.5 and 10.6 into Eq. 10.4 and dropping the fourth term in Eq. 10.4 since it is negligitle compared to the other terms we obtain the following form of the Continuity Equation:

$$
c \frac{\partial p}{\partial t}=-\frac{v}{r}-\frac{\partial v}{\partial r}
$$

Next we consider the Navier Stokes equations.

\subsection{Navier Stokes Equations}

Due to the geometry of the model the problem depends on the radial direction only. Since $v_{\theta}$ and $v_{z}$ are negligible the following simplified form of the Navier Stokes Equation is appropriate: 


$$
\begin{aligned}
\rho\left[\frac{D v_{r}}{D t}-\frac{v_{\theta}^{2}}{r}\right]= & -\frac{\partial p}{\partial r}+F_{r}+\mu\left[\nabla^{2} v_{r}-\frac{v_{r}}{r^{2}}-\frac{2}{r^{2}} \frac{\partial}{\partial \theta}\left(v_{\theta}\right)\right]+ \\
& {\left[\xi+\frac{1}{3} \mu\right] \frac{\partial}{\partial r}(\nabla \cdot V) }
\end{aligned}
$$

Simplifying and replacing $v_{r}$ by $v$ :

$$
\begin{aligned}
\rho \frac{\partial v}{\partial t}+\rho v \frac{\partial v}{\partial r}= & -\frac{\partial p}{\partial r}+\mu \frac{\partial^{2} v}{\partial r^{2}}+ \\
& \frac{\mu}{r} \frac{\partial v}{\partial r}+\frac{\mu}{r^{2}} \frac{\partial v}{\partial \theta}+\mu \frac{\partial^{2} v}{\partial z^{2}}
\end{aligned}
$$

Keeping only significant terms we obtain the final dimensional form of the Navier Stokes Equation:

$$
\rho \frac{\partial v}{\partial t}=-\frac{\partial p}{\partial r}+\mu \frac{\partial^{2} v}{\partial z^{2}}
$$

We solve the Navier Stokes and the Continuity equations simultaneously.

\subsection{Simultaneous Solution of System Equa- tions}

The governing equations are:

(a) The Navier Stokes Equation:

$$
\rho \frac{\partial v}{\partial t}=-\frac{\partial p}{\partial r}+\mu \frac{\partial^{2} v}{\partial z^{2}}
$$

(b) The Continuity Equation:

$$
\Omega c \frac{\partial p}{\partial t}=-\frac{v}{r}-\frac{\partial v}{\partial r}
$$


We make the following assumptions:

(a) We assume a velocity profile for fluid flow between two parallel plates.

(b) We average both equations along the $z$ direction since we assume that there is no flow along that direction.

The first assumption is the use of the following equation for fluid flow between two parallel plates.

$$
\begin{aligned}
& v(z)=\frac{6 z}{b}\left(1-\frac{z}{b}\right) v \\
& \frac{\partial v}{\partial z}=\left(\frac{6}{b}-\frac{12 z}{b^{2}}\right) v
\end{aligned}
$$

The second assumption allows us to average the equations along the $z$ direction. The Navier stokes Eq.10.11 becomes:

$$
\rho \frac{\partial \bar{v}}{\partial t}=-\frac{\partial \bar{p}}{\partial r}+\mu\left(\left.\frac{\partial \bar{v}}{\partial z}\right|_{z=b}-\left.\frac{\partial \bar{v}}{\partial z}\right|_{z=0}\right)
$$

or:

$$
\begin{array}{r}
\rho \frac{\partial \bar{v}}{\partial t}=-\frac{\partial \bar{p}}{\partial r}+\frac{\mu}{b}\left(-\frac{6}{b}-\frac{6}{b}\right) \bar{v} \\
\rho \frac{\partial \bar{v}}{\partial t}=-\frac{\partial \bar{p}}{\partial r}-\frac{12 \mu}{b^{2}} \bar{v}
\end{array}
$$

Differentiating with respect to $t$ :

$$
\rho \frac{\partial^{2} \bar{v}}{\partial t^{2}}=-\frac{\partial^{2} \bar{p}}{\partial r \partial t}-\frac{12 \mu}{b^{2}} \frac{\partial \bar{v}}{\partial t}
$$

Similarly averaging Eq. 10.12:

$$
\Omega c \frac{\partial \bar{p}}{\partial t}=-\frac{\bar{v}}{r}-\frac{\partial \bar{v}}{\partial r}
$$

Differentiating Eq. 10.17 with respect to $r$ :

$$
-\Omega c \frac{\partial^{2} \bar{p}}{\partial r \partial t}=\frac{1}{r} \frac{\partial \bar{v}}{\partial r}-\frac{1}{r^{2}} \bar{v}+\frac{\partial^{2} \bar{v}}{\partial r^{2}}
$$


Combining the two equations by substituting the value of the cross derivative from Eq. 10.16:

$$
\rho \Omega c \frac{\partial^{2} \bar{v}}{\partial t^{2}}=\frac{1}{r} \frac{\partial \bar{v}}{\partial r}-\frac{1}{r^{2}} \bar{v}+\frac{\partial^{2} \bar{v}}{\partial r^{2}}-\frac{12 \mu \Omega c}{b^{2}} \frac{\partial \bar{v}}{\partial t}
$$

We convert this equation into a non-dimensional form so that it will be easier to manipulate. The following dimensionless groups will be used:

$$
\begin{aligned}
p_{D} & =\frac{b^{2}}{12 \mu V L} p \\
v_{D} & =\frac{v}{V} \\
r_{D} & =\frac{r}{L} \\
t_{D} & =\frac{c_{s}}{L} t=\frac{t}{L \sqrt{\rho \Omega c}}
\end{aligned}
$$

Substituting Eqs 10.20-10.23 into Eq. 10.19:

$$
\frac{\partial^{2} \bar{v}_{D}}{\partial t_{D}^{2}}=\frac{1}{r_{D}} \frac{\partial \bar{v}_{D}}{\partial r_{D}}-\frac{\bar{v}_{D}}{r_{D}^{2}}+\frac{\partial^{2} \bar{v}_{D}}{\partial r_{D}^{2}}-\left(\frac{12 \mu L}{\rho b^{2} c_{s}}\right) \frac{\partial \bar{v}_{D}}{\partial t_{D}}
$$

Let:

$$
\beta=\frac{12 \mu L}{\rho b^{2} c_{\text {s }}}
$$

Then:

$$
\frac{\partial^{2} \bar{v}_{D}}{\partial t_{D}{ }^{2}}+\beta \frac{\partial \bar{v}_{D}}{\partial t_{D}}=\frac{1}{r d} \frac{\partial \bar{v}_{D}}{\partial r_{D}}+\frac{\partial^{2} \bar{v}_{D}}{\partial r_{D}^{2}}-\frac{\bar{v}_{D}}{r_{D}^{2}}
$$

We solve this equation by using the Laplace Transformation. Take the Laplace Transform of Eq. 10.26 and use the following notation: 


$$
L(\bar{v})=\hat{v_{D}}=\int_{0}^{\infty} e^{-s t} \bar{v}_{D}(t) d t
$$

Also the subscript $r$ and $r r$ will denote the first and second derivative with respect to $r$. Then:

$$
\hat{v_{D}}\left(s^{2}+\beta s\right)=\left(\hat{v_{D}}\right)_{r r}+\frac{1}{r_{D}}\left(\hat{v_{D}}\right)_{r}-\frac{\hat{v_{D}}}{r_{D}^{2}}
$$

or:

$$
r_{D}^{2}\left(\hat{v_{D}}\right)_{r r}+r_{D}\left(\hat{v_{D}}\right)_{r}-\hat{v_{D}}-r_{D}^{2} \hat{v_{D}}\left(s^{2}+\beta s\right)=0
$$

Substituting $\Lambda=s^{2}+\beta s$ we obtain an equation which resembles a Bessel Equation:

$$
r_{D}^{2}\left(\hat{v_{D}}\right)_{r r}+r_{D}\left(\hat{v_{D}}\right)_{r}-\hat{v_{D}}\left(1+\Lambda^{2} r_{D}^{2}\right)=0
$$

The solution of this equation is:

$$
\hat{v_{D}}\left(r_{D}, s\right)=C_{1} I_{1}\left(\Lambda r_{D}\right)+C_{2} K_{1}\left(\Lambda r_{D}\right)
$$

In order to obtain the full solution we determine the two constants $C_{1}$ and $C_{2}$ and invert the resulting expression to real space.

The Boundary conditions that govern the solution is:

$$
\begin{array}{r}
\hat{v_{D}}\left(r_{D}=\frac{r_{w}}{L}, s\right)=V(s) \\
\hat{v_{D}}(1, s)=0
\end{array}
$$


where $V(s)$ is some specified value for the velocity (in laplace space) at the mouth of the fracture where it meets the wellbore. Solving Eq. 10.30 for the boundary conditions Eq.10.34:

$$
\begin{aligned}
& C_{1}=-\frac{V(s) K_{1}(\Lambda)}{I_{1}(\Lambda) K_{1}\left(\Lambda \frac{\tau_{\mu}}{L}\right)-K_{1}(\Lambda) I_{1}\left(\Lambda \frac{\tau_{\mu}}{L}\right)} \\
& C_{2}=\frac{V(s) I_{1}(\Lambda)}{I_{1}(\Lambda) K_{1}\left(\Lambda \frac{r_{\omega}}{L}\right)-K_{1}(\Lambda) I_{1}\left(\Lambda \frac{r_{\mu}}{L}\right)}
\end{aligned}
$$

Having determined $C_{1}$ and $C_{2}$ we have fully determined the velocity of the fluid flow in the fracture but we have yet to determine the pressure response. To determine the pressure response in laplace space we use the continuity equation 10.17 which relates the two variables. Rewriting this:

$$
\Omega c \frac{\partial \bar{p}}{\partial t}=-\frac{\bar{v}}{r}-\frac{\partial \bar{v}}{\partial r}
$$

or in dimensionless form :

$$
\beta \frac{\partial \bar{p}_{D}}{\partial t_{D}}=-\frac{\bar{v}_{D}}{r_{D}}-\frac{\partial \bar{v}_{D}}{\partial r_{D}}
$$

Taking Laplace transform of this equation:

$$
s \beta \hat{p_{D}}=-\frac{\hat{v_{D}}}{r_{D}}-\frac{\partial \hat{v_{D}}}{\partial r_{D}}
$$

or

$$
\hat{p_{D}}=\frac{1}{s \beta}\left(-\frac{\hat{v_{D}}}{r_{D}}-\frac{\partial \hat{v_{D}}}{\partial r_{D}}\right)
$$

The solution for the velocity $\bar{v}_{D}$ in Laplace space from Eq. 10.30 is:

$$
\hat{v_{D}}\left(r_{D}, s\right)=C_{1} I_{1}\left(\Lambda r_{D}\right)+C_{2} K_{1}\left(\Lambda r_{D}\right)
$$


where $C_{1}$ and $C_{2}$ are known constants. We will differentiate the solution with respect to $r$ and substitute the result into the Eq. 10.35 which relates the velocity $\hat{v_{D}}$ to $\hat{p_{D}}$. Thus:

$$
\frac{\partial \hat{v_{D}}}{\partial r_{D}}=C_{1} \frac{\partial I_{1}\left(\Lambda r_{D}\right)}{\partial r_{D}}+C_{2} \frac{\partial K_{1}\left(\Lambda r_{D}\right)}{\partial r_{D}}
$$

Substituting Eq. 10.36 into Eq. 10.35:

$$
\begin{aligned}
\hat{p_{D}}\left(r_{D}, s\right) & =-\left(\frac{\Lambda}{s \beta}\right)\left(C_{1} I_{0}\left(\Lambda r_{D}\right)+C_{2} K_{0}\left(\Lambda r_{D}\right)\right) \\
C_{1} & =-\frac{V(s)}{I_{1}(\Lambda) K_{1}\left(\Lambda \frac{r_{w}}{L}\right)-K_{1}(\Lambda)} \\
C_{2} & =\frac{V(s) I_{1}(\Lambda)}{I_{1}(\Lambda) K_{1}\left(\Lambda \frac{r_{w}}{L}\right)-K_{1}(\Lambda) I_{1}\left(\Lambda \frac{r_{W}}{L}\right)}
\end{aligned}
$$

This solution may be evaluated for different values of $\beta$ by numerically inverting the solution in complex space using the crump algorithm. 


\section{Bibliography}

[1] Cooper H.H. Bredehoeft, J.L. and I.S. Papadopulos:, "Inertial and Storage Effects in Well-Aquifer Systems", Water Resources Research, (1966).

[2] Cooper H.H. Bredehoeft, J.R. and I.S. Papadopulos:, "The Response of Well-Aquifer Systems to Seismic Waves", Journal Of Geophysical Research, (1965).

[3] J.L. Bredehoeft:, "Response of Well-Aquifer Systems to Earth Tides", Journal of Geophysical Research, (1967).

[4] H.H. Cooper, Bredehoeft J.D, and Papadopulos I.S.:, "Response of a Finite Diameter Well to an Instantaneous charge of water", Water Resources Research, (1967).

[5] K.S. Crump:, "Numerical Inversion of Laplace Transforms using a Fourier Series Approximation", J.ACM, 23(1):89-96, (January 1976).

[6] Matthews C.S and Russell D.G.:, Pressure Buildup and Flow Tests in Wells, SPE of AIME, New York., (1967).

[7] H. Darcy:, Les Fontaines Publiques de la Ville de Dijon, Determination des lois d'ecoulement de l'eau a travers le sable, Victor Dalmont, France, (1856).

[8] T.M. Whittle D.Bourdet, J.A. Ayob:, "Interpreting Well Tests in fractured reservoirs", World Oil, (1983).

[9] D.W Dearholt and McSpadden:, Electromagnetic Wave Propagation, McGraw Hill, (1973). 
[10] McMillen, Foster and Odeh:, "The Equations of Motion of Fluids in Porous Media", Society Of Petroleum Engineers, (1967).

[11] J.K. Tan G.K. Nathan and K.C. Ng:, "Two-Dimensional analysis of Pressure Transients in Pipelines", International Journal for Numerical Methods in Fluids, (1987).

[12] R.B Guenther and Elizabeth Lundy:, "Well Response Tests", Inverse Problems 3, (1987).

[13] G.R. Holzhausen and H.N. Egan:, "Fracture Diagnostics in East Texas and Western Colorado using the Hydraulic impedanse method", SPE 15215, presented at the Unconventional Gas Technology Symposium held in, Applied Geomechanics Inc., 1986.

[14] G.R. Holzhausen and R.P. Gooch:, "Impedance Of Hydraulic Fractures", SPE 13892, presented at the 1985 Low Permeability Gas reservoirs held in, Colorado, 1985.

[15] Saldana Cortez M:, Drillstem Test Data Analysis Inertial and frictional wellbore effeects, $\mathrm{PhD}$ thesis, Stanford University, (1983).

[16] M.A. Saldana and H.J. Ramey Jr.:, "Slug Test and Drill Stem Test Flow Phenomena Including Wellbore Inertial and Frictional Effect", SPE 15118, presented at the 56th California Regional Meeting held in, Oakland, California., 1986.

[17] K. Shinohara and Ramey H. Jr.:, "Slug Test Data Analysis, Including the Inertial Effect of the Liquid in the Wellbore", presented at the 4th Annual Fall Technical Conference of the SPE held in Las Vegas, 1979.

[18] H. Stehfest:, "Algorithm 368, Numerical Inversion of the Laplace Transform", Communications of the ACM, (1970).

[19] Streeter and Wylie:, Fluid Mechanics, McGraw Hill Book Company, (1975).

[20] G. van der Kamp:, "Determining Aquifer Transmissivity by means of a Well Response Test: The Underdamped Case", Water Resources Research, 
(1976).

[21] E.Benjamin Wylie and V.Streeter:, Fluid Transients, FEB Press, (1982). 

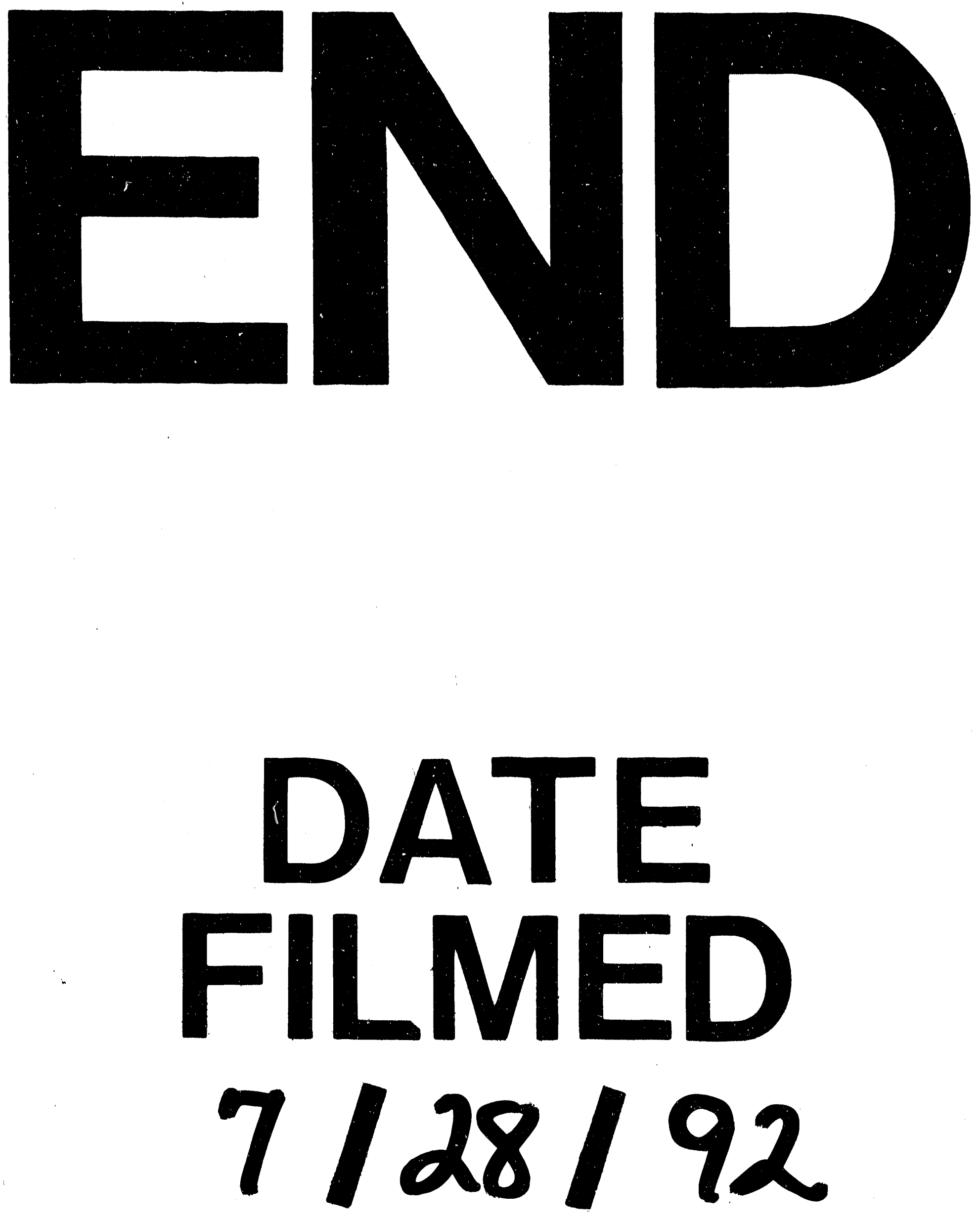
